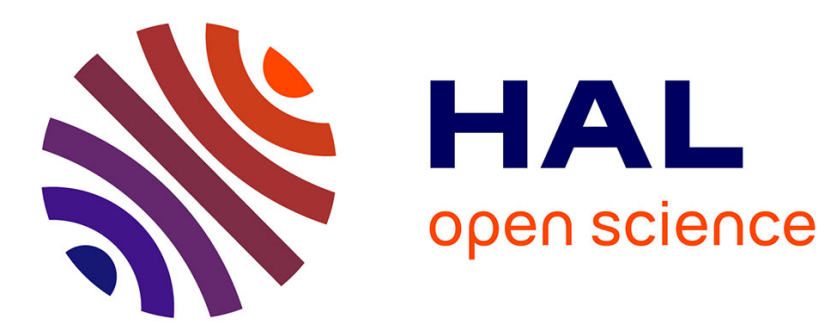

\title{
PrEP als demokratische Biopolitik. Zur Kritik der biopolitischen Repressionshypothese - oder: die pharmazeutische Destigmatisierung des Schwulseins.
}

Karsten Schubert

\section{- To cite this version:}

Karsten Schubert. PrEP als demokratische Biopolitik. Zur Kritik der biopolitischen Repressionshypothese - oder: die pharmazeutische Destigmatisierung des Schwulseins.. Jahrbuch Sexualitäten 5, 2020, pp.91-125. 10.6094/UNIFR/166195 . hal-02921471

\section{HAL Id: hal-02921471 \\ https://hal.science/hal-02921471}

Submitted on 25 Aug 2020

HAL is a multi-disciplinary open access archive for the deposit and dissemination of scientific research documents, whether they are published or not. The documents may come from teaching and research institutions in France or abroad, or from public or private research centers.
L'archive ouverte pluridisciplinaire HAL, est destinée au dépôt et à la diffusion de documents scientifiques de niveau recherche, publiés ou non, émanant des établissements d'enseignement et de recherche français ou étrangers, des laboratoires publics ou privés. 
www.karstenschubert.net

Schubert, Karsten (2020): PrEP als demokratische Biopolitik. Zur Kritik der biopolitischen Repressionshypothese - oder: die pharmazeutische Destigmatisierung des Schwulseins. In: Jahrbuch Sexualitäten 5, S. 91-125.

Accepted Authors Manuscript - Schreiben Sie mir gerne für die publizierte Version

PrEP als demokratische Biopolitik

Zur Kritik der biopolitischen Repressionshypothese - oder: die pharmazeutische Destigmatisierung des Schwulseins

Karsten Schubert

\section{Einführung: PrEP und Biopolitik ${ }^{1}$}

PrEP (Präexpositionsprophylaxe) ist ein relativ neues Mittel zur Prävention von HIV-Infektionen. HIV-negative Menschen nehmen antivirale Medikamente ein, die verhindern, dass der Kontakt mit dem Virus zu einer Infektion führt. Im Gegensatz zum Kondomgebrauch basiert dieses Präventionsverfahren auf Medikamenten und nicht auf einerVerhaltensänderung. Aus der Perspektive der Biopolitik fügt sie sich in einen größeren Trend in Richtung Medikalisierung, des Anstiegs der Macht der Pharmaindustrie und der Reglementierung des Risikos ein. Biopolitik bezeichnet in der Tradition Michel Foucaults die zunehmende Steuerung des Lebens durch die Politik mit Hilfe diverser Humanwissenschaften, wie beispielsweise der Medizin, der Sexualwissenschaft, derVolkswirtschaftslehre und der Demographie. Aus dieser Perspektive stellt die Erfindung dieser neuen Technologie neue Anforderungen an den Einzelnen - als solche ist sie nach Foucault eine Form der Macht. Die Hauptzielgruppe von PrEP sind schwule Männer, die eine hohe HIV-Prävalenz haben, wobei die

\footnotetext{
${ }^{1}$ Der vorliegende Artikel ist eine überarbeitete und aktualisierte Übersetzung von Karsten Schubert: The Democratic Biopolitics of PrEP. In: Helene Gerhards/Kathrin Braun (Hg.): Biopolitiken - Regierungen des Lebens heute. Wiesbaden 2019, S. 121-153. Ich danke Jon Catlin, V. Hunter Capps, Kathrin Braun, Helene Gerhards, David Halperin, Eloise Harris, Emmanuel Danan und Daniel Putnam für Kritik, Kommentare und Bemerkungen, die enorm dazu beigetragen haben, die Argumentation dieses Artikels zu entwickeln. Michèle Milde, Tabea Heppner, Josefine Morgan und Susanne Schlatter danke ich für die Hilfe bei der Übersetzung und das Lektorat der deutschen Version.
} 
Frage, welcher Untergruppe von schwulen Männern die Einnahme von PrEP empfohlen werden sollte, Gegenstand medizinischer Debatten ist. Sexuelles Verhalten ist das Ergebnis der Subjektivierung, des Prozesses, durch den soziale Normen Subjekte und ihre Wünsche bilden. Die Medikalisierung von schwulem Sex durch PrEP verändert die sexuelle Subjektivierung und geht daher mit einerVeränderung der Praktiken von schwulem Sex einher. Wo das Kondom das unbestrittene Mittel für Safer Sex war, ist es im Zeitalter von PrEP - zumindest im Blick auf HIV - technisch nicht mehr notwendig.Viele Schwule nutzen die neue Möglichkeit, sich auf kondomlosen und sicheren Sex einzulassen. Aber je mehr Menschen PrEP nehmen, desto mehr ändert sich die sexuelle Norm hin zu kondomlosem Sex, was den Einzelnen unter Druck setzen könnte, PrEP zu nehmen.

Nach einer kurzen Erläuterung der medizinischen Besonderheiten und der Geschichte von PrEP (1) vereint dieser Artikel eine Rekonstruktion zeitgenössischer theoretischer Ansätze zur Biopolitik im Zusammenhang mit PrEP (2) mit einer Darstellung aktueller politischer Debatten um PrEP (3). Die Untersuchung zeigt, dass sich Gouvernementalitätsstudien und die Italienische Theorie ${ }^{2}$ auf repressive Machtverhältnisse konzentrieren und daher die Komplexität der Debatte nicht berücksichtigen können, die sich aus der Subjektivität und Handlungsfähigkeit verschiedener beteiligter Akteure, insbesondere schwuler PrEP-Aktivisten, ergibt. Dennoch werden Interpretationen von PrEP als repressiver Macht auch von einigen Akteuren in der politischen Debatte um PrEP genutzt, meist ohne ausdrücklichen Bezug zur biopolitischen Theorie. Nikolas Roses und Paul Rabinows differenziertes Konzept der Biopolitik bietet einige Ansätze wie diejenigen der biopolitischen Bürgerschaft und der somatischen Ethik, um die Debatte um PrEP und die damit verbundenen komplexen Verhandlungen über Sexualität zu beschreiben. Ausgehend von diesen Ideen entwickle ich ein neues Konzept der ,demokratischen Biopolitik' von PrEP, die eine weitere Demokratisierung der Sexualität im biopolitischen Zeitalter ermöglicht. Der Artikel kritisiert so die ,biopolitische Repressionshypothese', also die Konzentration auf repressive Biomacht unter Ausblendung von demokratischen Elementen in weiten Teilen der biopolitischen und gouvernementalitätsanalytischen Debatte. Das Konzept der ,demokratischen Biopolitik‘ ist über PrEP hinaus ein Beitrag zu dieser allgemeinen Debatte um Biopolitik und um Foucaults Machtbegriff, denn es hilft bei der Überwindung der ,biopolitischen Repressionshypothese ${ }^{6}$ und ermöglicht es, Biopolitik differenzierter zu

\footnotetext{
${ }^{2}$,Italienische Theorie‘ ist ein Label für zeitgenössische italienische Ansätze der politischen Theorie, vertreten durch Giorgio Agamben, Antonio Negri, Paolo Virno und Roberto Esposito, vgl. u. a. Matteo Pasquinelli: The so-called Italian Theory and the revolt of living knowledge, http://www.uninomade.org/italian-theory-en/ [letzter Zugriff am 2.1.2020].
} 
analysieren und zu kritisieren.

Darüber hinaus behandelt der Artikel PrEP und die damit verbundenen Probleme aus der Perspektive der queeren und schwulen Theorie und trägt so zur Erforschung der Subjektivität von Schwulen bei. Obwohl der Diskurs im Gesundheitssektor versucht, Identitätskategorien wegen ihrer gut dokumentierten ausschließenden und repressiven Wirkung zu vermeiden, verwende ich aus zwei Gründen meist den Begriff ,Schwule‘ und nicht ,Männer, die Sex mit Männern haben“ (MSM): erstens, weil ,homosexuelle‘ PrEP-Nutzer sich meist als schwul identifizieren, da ein Selbstverständnis, einem besonderen Risiko der Infektion mit HIV ausgesetzt zu sein meist motivierend ist, PrEP zu nehmen - ein Selbstverständnis, das mit schwuler Identität verbunden ist; zweitens, weil die Debatte um PrEP zum Teil eine politische Aushandlung über schwule Identität beinhaltet, d. h. eine Verhandlung darüber, was es bedeutet, schwul zu sein, und über eine ,gute“ schwule Sexualethik. ${ }^{3}$ Indem der Artikel die politische Debatte um PrEP abbildet und sowohl die Pro-PrEP- als auch die Anti-PrEP-Position berücksichtigt, zeigt er, dass PrEP nicht nur zur HIV-Prävention, sondern auch zur Bekämpfung von Homophobie und zurVerbesserung der Lebensqualität von Schwulen durch die Destigmatisierung von schwulem Sex beiträgt.

\footnotetext{
${ }^{3}$ Race ist, insbesondere in den USA, ein entscheidender Faktor für die HIV-Epidemie. Afroamerikaner haben eine höhere HIV-Rate als andere Ethnien und Weiße, wobei Schwarze MSM die verletzlichste Gruppe sind: „Gay and bisexual men continue to be most affected by the HIV epidemic in the U.S. At current rates, 1 in 6 MSM will be diagnosed with HIV in their lifetime, including 1 in 2 black MSM, 1 in 4 Latino MSM, and 1 in 11 white MSM. African Americans are by far the most affected racial or ethnic group with a lifetime HIV risk of 1 in 20 for men (compared to 1 in 132 for whites) and 1 in 48 for women (compared to 1 in 880 for whites) ", Centers for Desease Control and Prevention: Lifetime Risk of HIV Diagnosis. Half of black gay men and a quarter of Latino gay men projected to be diagnosed within their lifetime, https://www.cdc.gov/nchhstp/newsroom/2016/croi-press-releaserisk.html [letzter Zugriff am 2.1.2020]. Viele Schwarze amerikanische MSM identifizieren sich nicht als schwul oder bisexuell, da die schwule Mainstream-Kultur der USA überwiegend Weiß ist. Die komplexen Gründe für die extreme Epidemie bei Schwarzen Schwulen oder bei sich als nicht schwul identifizierenden MSM und die schwierigen Versuche, PrEP in ihre Gemeinschaften zu einzuführen, gehen über den Rahmen dieses Artikels hinaus, vgl. Linda Villarosa: America's Hidden H.I.V. Epidemic. Why do America's black gay and bisexual men have a higher H.I.V. rate than any country in the world? In: New York Times Magazine, 2017, https://www.ny-

times.com/2017/06/06/magazine/americas-hidden-hiv-epidemic.html [letzter Zugriff am 2.1.2020). Mit seinem Fokus auf die Debatte des schwulen Mainstreams im globalen Norden wird vorliegender Artikel faktisch von Weißen Perspektiven dominiert.
} 


\section{PrEP - Die Medikalisierung der HIV-Prävention}

Studien zeigen, dass PrEP hochwirksam ist, mit einem Schutzniveau von etwa $92 \%$, wenn täglich eine Pille der Wirkstoffkombination Emitricitabin und Tenofovir (Handelsname Truvada) eingenommen wird. ${ }^{4}$ Hier kann also eine höhere Schutzwirksamkeit erzielt werden als bei Kondomen, dem klassischen verhaltensbezogenen Präventionsverfahren, dessen Schutzwirksamkeit bei MSM bei etwa $70 \%$ liegt. ${ }^{5}$ PrEP ist nicht unbedingt eine vollständige Alternative zu verhaltensbasierten Präventionsmethoden wie Kondomgebrauch oder Serosorting, ${ }^{6}$ und wird teilweise parallel zu diesen Methoden als zusätzliches Präventionsmittel eingesetzt. Dies liegt insbesondere daran, dass PrEP nur vor HIV, nicht aber vor anderen Sexuell übertragbaren Infektionen (STIs) schützt. Derzeit ist Truvada das einzige Medikament, das für die PrEP-Anwendung zugelassen ist, aber andere orale Medikamente wie Descovy ${ }^{7}$ und Formen wie Vaginalgel werden derzeit in Studien getestet. Truvada wurde auch in einem „On Demand“-Schema getestet, bei dem das Medikament erst kurz vor und nach einem Infektionsrisiko eingenommen wird, wobei die Schutzwirkung geringer ist als bei der täglichen Einnahme. ${ }^{8}$

\footnotetext{
${ }^{4}$ Die Schutzwirkung bezieht sich auf den Risikounterschied einer HIV-Übertragung pro Geschlechtsakt zwischen dem Verzicht auf Schutz und der Verwendung der jeweiligen Schutztechnologie (PrEP, Kondom, Serosorting etc.), vgl. Robert M. Grant/Javier R. Lama/Peter L. Anderson/Vanessa McMahan/Albert Y. Liu/Lorenza Vargas/Pedro Goicochea/Martín Casapía/Juan Vicente Guanira-Carranza/Maria E. Ramirez-Cardich/Orlando Montoya-Herrera/Telmo Fernández/Valdilea G. Veloso/Susan P. Buchbinder/Suwat Chariyalertsak/Mauro Schechter/Linda-Gail Bekker/Kenneth H. Mayer/Esper Georges Kallás/Rivet Amico/Kathleen Mulligan/Lane R. Bushman/Robert J. Hance/Carmela Ganoza/Patricia Defechereux/Brian Postle/Furong Wang/Jeff McConnell/Jia-Hua Zheng/Jeanny Lee/James F. Rooney/Howard S. Jaffe/Ana I. Martinez/David N. Burns/David V. Glidden: Preexposure Chemoprophylaxis for HIV Prevention in Men Who Have Sex with Men. In: New England Journal of Medicine 363 (2010), H. 27, S. 2587-2599; Sheena McCormack/David T. Dunn/Monica Desai/David I. Dolling/Mitzy Gafos/Richard Gilson/Ann K. Sullivan/Amanda Clarke/Iain Reeves/Gabriel Schembri/Nicola Mackie/Christine Bowman/Charles J. Lacey/Vanessa Apea/Michael Brady/Julie Fox/Stephen Taylor/Simone Antonucci/Saye H. Khoo/James Rooney/Anthony Nardone/Martin Fisher/Alan McOwan/Andrew N. Phillips/Anne M. Johnson/Brian Gazzard/Owen N. Gill: Pre-exposure prophylaxis to prevent the acquisition of HIV-1 infection (PROUD): effectiveness results from the pilot phase of a pragmatic open-label randomised trial. In: The Lancet 387 (2016), H. 10013, S. 5360; Christoph D. Spinner/Christoph Boesecke/Alexander Zink/Heiko Jessen/Hans-Jürgen Stellbrink/Jürgen Kurt Rockstroh/Stefan Esser: HIV pre-exposure prophylaxis (PrEP). A review of current knowledge of oral systemic HIV PrEP in humans. In: Infection 44 (2016), H. 2, S. 151-158.

${ }^{5}$ Vgl. Dawn K. Smith/Jeffrey H. Herbst/Xinjiang Zhang/Charles E. Rose: Condom Effectiveness for HIV Prevention by Consistency of Use Among Men Who Have Sex With Men in the United States. In: Journal of Acquired Immune Deficiency Syndromes 68 (2015), H. 3, S. 337-344; Benjamin Ryan: How Well Do Condoms and PrEP Prevent HIV Among Gay and Bi Men? CDC researchers have estimated how well condoms and PrEP prevent HIV in gay and bisexual men. How much faith can be placed in these figures?, 2015, https://www.poz.com/article/condom-PrEP-efficacy-26766-8889 [letzter Zugriff am 18.7.2018].

${ }^{6}$ Serosorting bedeutet die Auswahl von Sexualpartnern nach ihrem Serostatus, z. B. wenn eine HIV-positive Person Sex mit einem HIV-positiven Partner hat.

${ }^{7}$ Vgl. AVAC: DISCOVER Trial Factsheet, https://www.avac.org/discover-trial-factsheet [letzter Zugriff am 2.1.2020].

${ }^{8}$ Vgl. Jean-Michel Molina/Catherine Capitant/Bruno Spire/Gilles Pialoux/Laurent Cotte/Isabelle Charreau/Cecile
} 
Truvada stoppt die Vermehrung des HI-Virus in den Zellen einer HIV ausgesetzten Person, so dass diese Person nicht infiziert wird. Die Infektion wird dadurch bewirkt, dass viruseigene RNA in die DNA der infizierten Zelle kopiert wird. Dies erfolgt durch die Tätigkeit des Enzyms Reverse-Transkriptase. Danach produziert die infizierte Wirtszelle neue Viren. Tenofovir und Emitricitabin sind Reverse-Transkriptase-Inhibitoren (RTI), die diesen Reproduktionsprozess verhindern, indem sie die Enzyme verändern, die für die Kopie der RNA in die DNA der Wirtszelle erforderlich sind.

PrEP kann innerhalb eines allgemeinen Trends zur Medikalisierung von HIV-Prävention und Sexualität verortet werden. ${ }^{9}$ Klassische Prävention betraf das Verhalten und operierte durch Werbung für die Verwendung von Kondomen, den Verzicht auf bestimmte Sexualpraktiken oder auf Sex überhaupt. Im Gegensatz zur verhaltensbasierten Prävention minimiert die medizinische Prävention die Infektionsgefahr durch die Verabreichung von Medikamenten. ${ }^{10}$ Andere Technologien der medizinischen Prävention, die PrEP vorausgingen, sind „Schutz durch Therapie“ (Treatment as prevention, TasP) und Postexpositionsprophylaxe (PEP). ${ }^{11}$ TasP bedeutet die Senkung derViruslast von HIVpositiven Patienten durch antiretrovirale (ARV) Medikamente, so dass sie nicht mehr infektiös sind. PEP bezieht sich auf ein Notfallverfahren mit ARV-Medikamenten nach einer (potenziellen) Exposition, das unmittelbar nach der Exposition beginnen muss, um effizient zu sein, und im Gegensatz zu PrEP aufgrund einer anderen Medikamentenkombination erhebliche Nebenwirkungen aufweist. Der entscheidende Unterschied zwischen verhaltensbezogener und medizinischer Prävention ist der

Tremblay/Jean-Marie Le Gall/Eric Cua/Armelle Pasquet/François Raffi/Claire Pintado/Christian Chidiac/Julie Chas/Pierre Charbonneau/Constance Delaugerre/Marie Suzan-Monti/Benedicte Loze/Julien Fonsart/Gilles Peytavin/Antoine Cheret/Julie Timsit/Gabriel Girard/Nicolas Lorente/Marie Préau/James F. Rooney/Mark A. Wainberg/David Thompson/Willy Rozenbaum/Veronique Doré/Lucie Marchand/Marie-Christine Simon/Nicolas Etien/Jean-Pierre Aboulker/Laurence Meyer/Jean-François Delfraissy: On-Demand Preexposure Prophylaxis in Men at High Risk for HIV-1 Infection. In: The New England Journal of Medicine 373 (2015), H. 23, S. 2237-2246; Sophie Cousins: PrEP on demand reduces HIV infections, study finds. In: BMJ 358 (2017), j3619.

${ }_{9} \mathrm{Vgl}$. Thea Cacchioni/Leonore Tiefer: Why Medicalization? Introduction to the Special Issue on the Medicalization of Sex. In: Journal of sex research 49 (2012), H. 4, S. 307-310.

${ }^{10} \mathrm{Vgl}$. Alain Giami/Christophe Perrey: Transformations in the Medicalization of Sex: HIV Prevention between Discipline and Biopolitics. In: Journal of Sex Research 49 (2012), H. 4, S. 353-361.

${ }^{11} \mathrm{Vgl}$. Myron S. Cohen/Marybeth McCauley/Theresa R. Gamble: HIV treatment as prevention and HPTN 052. In: Current Opinion in HIV and AIDS 7 (2012), H. 2, S. 99-105; Andrew D. Forsyth/Ronald O. Valdiserri: Reaping the prevention benefits of highly active antiretroviral treatment: policy implications of HIV Prevention Trials Network 052. In: Current Opinion in HIV and AIDS 7 (2012) H. 2, S. 111-116; Myron S. Cohen/M. Kumi Smith/Kathryn E. Muessig/Timothy B. Hallett/Kimberly A. Powers/Angela D. Kashuba: Antiretroviral treatment of HIV-1 prevents transmission of HIV-1: where do we go from here? In: The Lancet 382 (2013), H. 9903, S. 15151524; Binta Sultan/Paul Benn/Laura Water: Current perspectives in HIV post-exposure prophylaxis. In: HIV/AIDS 6 (2014), S. 147-158. 
Zeitpunkt der Präventionsmaßnahme.Verhaltensbasierte Prävention erfordert eine präventive Entscheidung während der sexuellen Aktivität, wohingegen bei der medizinischen Prävention der bewusste Präventionsakt (Einnahme einer Pille) vom sexuellen Akt entkoppelt ist.

Truvada wurde erstmals 2012 von der Food and Drug Administartion (FDA) in den USA für den Einsatz als PrEP zugelassen und über private Krankenversicherungen verbreitet, wodurch wirtschaftlich Privilegierte einen einfachen und Menschen ohne ausreichende Gesundheitsabsicherung einen schwierigen Zugang dazu haben. In Europa waren die öffentlichen und privaten Krankenversicherungen langsamer bei der Übernahme von PrEP. Die Kosten von rund 900 Euro pro Monat stellten ein entscheidendes Problem dar, bis das Patent von Truvada im Juli 2017 in den meisten europäischen Ländern abgelaufen war. ${ }^{12}$ Länder des globalen Südens, insbesondere Indien, produzieren seit vielen Jahren Generika von Truvada und anderen HIV-Medikamenten und gehen juristisch gegen restriktive Auslegungen des Patentschutzes vor, um die HIV-Epidemien in ihren Ländern zu bekämpfen.Viele europäische Schwule, für die PrEP bis vor kurzem nicht durch ihr Gesundheitssystem abgedeckt war, bestellten billige Truvada-Generika aus Indien oder Thailand und benutzten diese oft ohne professionelle Betreuung. In den letzten drei Jahren haben die Krankenversicherungen aller westeuropäischen Länder mit Ausnahme Österreichs angefangen, die PrEP als Kassenleistung zu übernehmen. In Deutschland ist dies seit September 2019 der Fall.

\section{Die biopolitische Perspektive}

Biopolitik ist ein von Michel Foucault geprägtes Konzept, das er in „Sexualität und Wahrheit“ und der „Geschichte der Gouvernementalität“"13 entwickelt. Foucault argumentiert hier, dass die moderne Gouvernementalität durch eine bestimmte Form von Macht über das Leben funktioniere,

\footnotetext{
${ }^{12}$ Vgl. Pascale Boulet: Will the European Court of Justice put a stop to the evergreening of Truvada patents?, 2018, https://medicineslawandpolicy.org/2018/05/will-the-european-court-of-justice-put-a-stop-to-the-evergreening-oftruvada-patents/ [letzter Zugriff am 21.8.2018]; Medical Express: EU door opens for generic version of AIDS medicine Truvada, 2018, https://medicalxpress.com/news/2018-07-eu-door-version-aids-medicine.html [letzter Zugriff am 21.8.2018]. In den Vereinigten Staaten ist das Patent auf Truvada noch geschützt, und erschwingliche Generika sind noch nicht verfügbar, vgl. Keren Landman: The FDA Has Approved Generic PrEP - but Access May Remain Difficult, 2017, https://www.vice.com/en_us/article/xw875w/the-fda-has-approved-generic-prepbut-access-mayremain-difficult [letzter Zugriff am 21.8.2018].

${ }^{13}$ Michel Foucault: Der Wille zum Wissen. Sexualität und Wahrheit 1. Übersetzt von Ulrich Raulff und Walter Seitter. Frankfurt a. M. 1983 [zuerst frz. 1976]; ders.: Geschichte der Gouvernementalität I. Sicherheit, Territorium, Bevölkerung. Vorlesung am Collège de France 1977-1978. Hg. von Michel Sennelart. Übersetzt von Claudia Brede-
} 
welche sowohl das Individuum als auch das Kollektiv regiert. Gouvernementalität ist ein Konzept, dass die moderne Art des Regierens beschreibt, die auf humanwissenschaftlichem Wissen beruht, beispielsweise über Sexualität, Psychologie, Demograhie und Statistik. Auf individueller Ebene operiert die Biopolitik durch disziplinäre Macht, welche Foucault in seinem früheren Werk „Überwachen und Strafen“"14 analysiert. Auf der kollektiven Ebene ist Biopolitik die Regulierung der Bevölkerung durch wissenschaftliche Erkenntnisse, beispielsweise wenn versucht wird, die Geburtenraten zu erhöhen, indem die medizinische Überwachung von Frauen intensiviert wird und Sexualität, die nicht zur Reproduktion beiträgt, pathologisiert wird. Die Biopolitik verbinde diese beiden Machtebenen: „Die Disziplinen des Körpers und die Regulierungen der Bevölkerung bilden die beiden Pole, um die herum sich die Macht zum Leben organisiert hat. " ${ }^{15}$ Foucault unterscheidet Biopolitik oder Biomacht ${ }^{16}$ von souveräner Macht. Während souveräne Macht durch das „Recht, sterben zu machen oder leben zu lassen“ gekennzeichnet sei, komme der Biomacht zusätzlich das biopolitische „Recht, Leben zu machen und sterben zu lassen“ zu. ${ }^{17}$ Diese Differenzierung ist eine Beschreibung des historischen Wandels hin zur modernen Gouvernementalität. Die alte, souveräne Macht hatte das Recht, individuelle Leben zu nehmen, und ließ die individuellen Leben ansonsten allein und unreguliert (,leben lassen'). Die moderne Biopolitik ist eine Erweiterung und Intensivierung der Macht, insofern sie versucht, die Bevölkerung produktiv zu regulieren und damit viele Aspekte des täglichen Lebens zu regeln, die außerhalb des Fokus der souveränen Macht lagen, wie Sexualität, Gesundheit und Lebensweise.

Foucaults Konzept der Biopolitik erwies sich als enorm produktiv und beflügelte die Entwicklung ganzer Forschungsgebiete. ${ }^{18} \mathrm{Um}$ PrEP durch eine biopolitische Linse kritisch zu analysieren, schlage

Konersmann und Jürgen Schröder. Frankfurt a. M. 2004 [zuerst frz. 2004]; ders.: Geschichte der Gouvernementalität II. Die Geburt der Biopolitik. Vorlesung am Collège de France 1978-1979. Hg. von. Michel Sennelart. Übersetzt von Jürgen Schröder. Frankfurt a. M. 2006 [zuerst frz. 2004].

${ }^{14}$ Michel Foucault: Überwachen und Strafen. Die Geburt des Gefängnisses. Übersetzt von Walter Seitter. Frankfurt a. M. 1994 [zuerst frz. 1975].

${ }^{15}$ Foucault: Wille (wie Anm. 13), S. 166. „Macht zum Leben“ ist hier synonym mit Biopolitik und Biomacht zu verstehen.

${ }^{16}$ Foucault unterscheidet nicht systematisch zwischen Biopolitik und Biomacht.

${ }^{17}$ Michel Foucault: In Verteidigung der Gesellschaft. Vorlesungen am Collège de France 1975-1976. Übersetzt von Michaela Ott. Frankfurt a. M. 1999 [zuerst frz. 1997], S. 284.

${ }^{18}$ Für einen allgemeinen Überblick siehe Thomas Lemke: Biopolitik zur Einführung. Hamburg 2007; Catherine Mills: Biopolitics. Abingdon 2018. Mike Laufenberg: Sexualität und Biomacht. Vom Sicherheitsdispositiv zur Politik der Sorge. Bielefeld 2014, bietet die beste Interpretation von Foucaults Biopolitiktheorie in Hinblick auf queere Sexualität. Laufenberg kritisiert dort auch die (heteronormative) Ausblendung der Sexualität in den etablierten biopolitischen Ansätzen. 
ich vor, die Debatte über Biopolitik wie folgt darzustellen. Die Biopolitik einerseits wird als evaluierendes Konzept der Sozialkritik und andererseits als differenzierte Analyse zeitgenössischer Entwicklungen verwendet. Die evaluierende Nutzung des Konzepts der Biopolitik kann in zwei Teilstränge unterteilt werden: Erstens ist die Biopolitik ein zentrales Konzept für Gouvernementalitätsstudien, die sich auf Foucaults nicht-normativen Ansatz der genealogischen Kritik ${ }^{19}$ stützen, um zeitgenössische Gouvernementalität zu analysieren und zu kritisieren. Zweitens bilden biopolitische Konzepte einen integralen Bestandteil der zeitgenössischen großen Erzählungen über die Moderne in der italienischen Theorie, vertreten durch Giorgio Agamben, Antonio Negri, Michael Hardt und Roberto Esposito. Die differenzierte Analyse der Biopolitik wird von Rose und Rabinow repräsentiert. Im Folgenden werde ich kurz auf diese drei Stränge eingehen, um Positionen zu PrEP zu rekonstruieren, die innerhalb ihrer jeweiligen Rahmenbedingungen artikuliert werden oder werden könnten. ${ }^{20}$

Gouvernementalitätsstudien. Gouvernementalität ist ein von Foucault geprägtes Konzept, das eng mit der Biopolitik verbunden ist. Die Gouvernementalität beschreibt drei miteinander verbundene Aspekte: erstens, die Form der modernen Regierung, deren Gegenstand die Bevölkerung ist (und die insofern als gleichbedeutend mit Biopolitik verstanden werden kann) und auf staatlichem Wissen und staatlichen Rationalitäten basiert; zweitens die historische Tendenz zu dieser Gouvernementalität im Westen; und drittens das Ergebnis dieses Prozesses, der moderne Verwaltungsstaat, der gouvernementalisiert wird. ${ }^{21}$ Genauer gesagt, ist Gouvernementalität ,liberale Gouvernementalität, und Foucault beschrieb ihre Genealogie bis zur Bildung des zeitgenössischen Neoliberalismus, in dem der Markt als natürliches Objekt betrachtet wird, der sich jedoch auf eine spezifische Subjektivierung und Regulierung stützt. ${ }^{22}$ Gouvernementalitätsanalysen knüpfen an Foucault an und untersuchen verschiedene Aspekte der neoliberalen Gouvernementalität mit dem Ziel, die repressiven Sei-

\footnotetext{
${ }^{19}$ Vgl. Martin Saar: Genealogie als Kritik. Geschichte und Theorie des Subjekts nach Nietzsche und Foucault. Frankfurt a. M./New York 2007.

${ }^{20}$ Die Bezeichnungen werden hier verwendet, um das Feld grob abzubilden. Die spezifischen Positionen zu HIV und PrEP, die ich als Teilstränge oder Schule bezeichne, erfassen die Positionen dieser Schulen nicht vollständig. Auch wenn die Argumente bezüglich PrEP, die ich rekonstruiere, typisch für die drei jeweiligen Schulen sind, könnten mit ihren komplexen Konzepten verschiedene Argumente konstruiert werden.

${ }^{21}$ Vgl. Foucault: Gouvernementalität I (wie Anm. 13), S. 162 f.

${ }^{22}$ Vgl. Foucault: Gouvernementalität II (wie Anm. 13).
} 
ten der neoliberalen Gouvernementalität und die von ihr hervorgebrachten Subjektivierungen aufzudecken. ${ }^{23}$ Wo die neoliberale Regierung Freiheit proklamiert, analysieren kritische Gouvernementalitätsstudien Normierung und Kontrolle durch Subjektivierung als eigenverantwortlich und rechenschaftspflichtig, was die Subjekte ermutigt, sich ihr ganzes Leben lang wie rationale homines economici zu verhalten. ${ }^{24}$

Während PrEP in der Gouvernementalitätsforschung bis jetzt kaum diskutiert wurde, ist Tim Deans Aufsatz „Mediated intimacies: Raw sex, Truvada, and the biopolitics of chemoprophylaxis“25 eines der wenigen Beispiele dafür, wie die kritische Hermeneutik von Gouvernementalitätsstudien zur Analyse von PrEP genutzt werden kann. Dean konzentriert sich auf die potenziell (oder tatsächlich) repressiven Effekte von PrEP und analysiert sie als Element umfassendererVeränderungen in der zeitgenössischen Gouvernementalität und Biopolitik, der Medikalisierung und dem Diskurs der Prävention, der die individuelle Verantwortung und rationale Entscheidungsfindung betont. Er fragt, was bei PrEP biopolitisch auf dem Spiel steht, und beschreibt sie als Aufforderung und sogar Imperativ der ,pharmazeutischen Macht“26 an Schwule, ihr Sexualleben zu ändern. ${ }^{27}$ PrEP, so Dean, sei ein typischer Fall von Biopolitik, die darauf abziele, ,zunehmend die Kontrolle über die Bevölkerung durch Gesundheits- und Wellnesspraktiken zu erlangen. [...] Biomacht überzeugt uns davon, dass es in unserem eigenen Interesse liegt, die Ernährung, Bewegung und Medikamenteneinnahme zu regulieren, um unsere allgemeine Gesundheit zu optimieren.“ “28 PrEP sei Teil der ,normalisierenden Gesundheitspolitik“" ${ }^{29}$ und beinhalte eine strenge Überwachung schwuler Körper durch medizinische Autoritäten, die laut Dean abgelehnt werden sollte, weil es sich um eine Regierungsform

\footnotetext{
${ }^{23}$ Vgl. Graham Burchell/Colin Gordon/Peter Miller (Hg.): The Foucault Effect. Studies in Governmentality. Chicago 1991; Mitchell Dean: Governmentality. Power and Rule in Modern Society. London 1999; Thomas Lemke: Gouvernementalität und Biopolitik. 2. Aufl. Wiesbaden 2008; Ulrich Bröckling/Susanne Krasmann/Thomas Lemke: From Foucault's Lectures at the Collège de France to Studies of Governmentality. An Introduction. In: diess. (Hg.), Governmentality. Current Issues and Future Challenges. New York/London 2011, S. 1-33; Jakob Nilsson/SvenOlov Wallenstein (Hg.): Foucault, Biopolitics, and Governmentality (Södertörn Philosophical Studies, Bd. 14). Huddinge 2013.

${ }^{24}$ Vgl. Ulrich Bröckling: Das unternehmerische Selbst. Soziologie einer Subjektivierungsform. 5. Aufl. Frankfurt a. M. 2013. Vgl. für eine Kritik am Antinormativismus in zeitgenössischen Regierungsstudien Karsten Schubert: Freiheit als Kritik. Sozialphilosophie nach Foucault. Bielefeld 2018, S. 104-111.

${ }^{25}$ Tim Dean: Mediated intimacies: Raw sex, Truvada, and the biopolitics of chemoprophylaxis. In: Sexualities 18 (2015) 1-2, S. 224-246.

${ }^{26}$ Alle Zitate aus englischen Quellen sind durch K.S. ins Deutsche übersetzt, wenn nicht anders vermerkt.

${ }^{27}$ Dean: Mediated (wie Anm. 25), S. 232.

${ }^{28}$ Ebd., S. 233.

${ }^{29}$ Ebd.
} 
handele: „Niemand will von einer Regierungsbehörde gesagt bekommen, wie er Sex haben darf.“30 Ausgehend vom Widerstand gegen PrEP in der schwulen Community argumentiert Dean, dass es „,biopolitische Nebenwirkungen (zusätzlich zu physiologischen) durch die massenhafte Einhaltung pharmazeutischer Regeln und Kontrollen hervorrufe, die berücksichtigt werden sollten. ${ }^{31}$

Die Kritik von Dean lässt sich in zwei Punkten zusammenfassen. Zunächst kritisiert er den dogmatisch-medizinischen Diskurs der HIV-Prävention, der auf Rationalität und Eigenverantwortung setzt: „Die mangelnde Bereitschaft, Sexualität als etwas Anderes als im Wesentlichen rationales Verhalten zu diskutieren, ist erstaunlich. Diese mangelnde Bereitschaft erhält ein Klima aufrecht, in dem sexuelle Aktivitäten, die nicht als Ausdruck des individuellen Eigeninteresses erscheinen, pathologisiert werden. “32 In diesem Paradigma, das im öffentlichen Gesundheitsdiskurs vielerorts hegemonial ist, fallen Begehren, Fantasie und Gemeinschaft aus dem Bild von Sex heraus, was zu unplausiblen Erzählungen über schwules Risikoverhalten führt. ${ }^{33}$ Aus der Sicht des epidemiologischen Gesundheitswesens kann Sex ohne Kondome nur im Rahmen von Drogenmissbrauch erklärt werden, da angeblich keine rational denkende Person jemals solche sexuellen Risiken eingehen würde. ${ }^{34}$ Die Dominanz biophysiologischer Erklärungen in der Wissenschaft und die Tatsache, dass nicht-naturwissenschaftliche, kulturelle Interpretationen von Sex in vielen Ländern aus dem Diskurs über die öffentliche Gesundheit ausgeschlossen sind, nützen laut Dean der Pharmaindustrie, da dies eine Nachfrage nach ihren Produkten schafft. Dies sei eine wirtschaftliche Intensivierung dessen, was Foucault scientia sexualis nannte. ${ }^{35}$

\footnotetext{
${ }^{30}$ Ebd.

${ }^{31}$ Ebd., S. 234 [Hervorhebung im Original].

${ }^{32}$ Ebd., S. 235.

${ }^{33}$ Dies trifft auf den deutschen HIV-Präventionsdiskurs nur eingeschränkt zu, der früh von einer ,großen Koalition“, die communitybasierte Ansätze unterstützt und institutionalisiert hat, geprägt wurde, vgl. Michael Bochow: Vom Safer Sex zur Präexpositionsprophylaxe (PrEP): Kurze Formeln für lange Wege. In: Patrick Henze/Aaron Lahl/Victoria Preis (Hg.): Psychoanalyse und männliche Homosexualität. Beiträge zu einer sexualpolitischen Debatte. Gießen 2019, S. 259-278.

${ }^{34}$ Für verschiedene Berichte über riskanten schwulen Sex siehe Tim Dean: Unlimited Intimacy. Reflections on the Subculture of Barebacking. Chicago 2009 (psychoanalytisch), und David M. Halperin: What Do Gay Men Want? An Essay on Sex, Risk, and Subjectivity. Ann Arbor 2007; ders.: The Biopolitics of HIV Prevention Discourse. In: Vernon W. Cisney/Nicolae Morar (Hg.): Biopower. Foucault and Beyond. Chicago/London 2016, S. 199-227 (gegen Psychoanalyse).

${ }^{35}$ Vgl. Foucault: Wille (wie Anm. 13), Kap. III. Siehe für eine Kritik an PrEP-Studien, die argumentiert, dass sie einem pharmazeutischen Paradigma folgten, das keine kulturellen Präventionsverfahren berücksichtigen könne Cindy Patton/Hye Jin Kim: The Cost of Science. Knowledge and Ethics in the HIV Pre-Exposure Prophylaxis Trials. In: Journal of Bioethical Inquiry 9 (2012), H. 3, S. 295-310.
} 
Zweitens greift Dean auf Paul Preciados „Testo Junkie“36 und sein Konzept von „Pharmamacht“37 zurück. Preciado vertritt die Position, dass Macht heutzutage auf der molekularen Ebene wirkt, durch die Pharmazie, die zu einem festen Bestandteil der Sexualität geworden ist: „Biomacht“, so Dean, ,dringt nicht nur durch psychologische Identifikationsmechanismen in uns ein (wenn wir herausfinden, wer wir in sexueller Hinsicht wirklich sind), sondern auch durch die Pharmazeutika, die wir einnehmen, um die sexuellen Wesen zu werden, die wir sein wollen. " 38 Preciado baut sein Argument zur „Pharmamacht“ auf, indem er einen Zusammenhang zwischen der Verhütungspille für Frauen und der Biotechnologie für trans` Menschen, wie z.B. Hormonen, herstellt, um zu zeigen, dass beide aus dem gleichen biopolitischen ,,industriellen Sex-Gender-Komplex“ stammen. ${ }^{39}$ Dieser Komplex verändere die Sexualität grundlegend, indem er sie technologisiere und eine neue Überwachung und Kontrolle ermögliche. Durch einen Rückgriff auf Pornographie, die Begehren erzeugt, ${ }^{40}$ habe Biomacht heute, so Dean, ,vollen Zugang zu unseren Körpern und deren Begehren im Dienste des wirtschaftlichen Profits. “" ${ }^{41}$ PrEP habe einen tiefgreifenden Einfluss auf schwule sexuelle Subjektivität und schwules Begehren und schaffe eine neue Lust auf kondomlosen Sex, die durch Pornografie, Pharmaindustrie und das Gesundheitswesen vermittelt werde. ${ }^{42}$ Daher ist PrEP laut Dean nur die nächste Intensivierung dieses ökonomisch getriebenen biopolitischen Regimes und eine neue Phase in der langen Geschichte der Medikalisierung der schwulen Sexualität. ${ }^{43}$

Biopolitik in großen Erzählungen der Moderne. Zeitgenössische italienische Politologen wie Agamben, Negri, Hardt und Esposito machen die Biopolitik zu einer kritischen Kategorie für die Analyse der Moderne, die sich lose an Foucault orientiert. In ihren Arbeiten ist die Biopolitik das bestimmende Merkmal der Moderne. Agamben zeichnet ein dunkles Bild der modernen Biomacht als absoluter Souveränität, die das biologische Leben kontrolliert (zoê im Gegensatz zu bios) und das vergesellschaftlichte (bios) auf das bloße Leben (zoê) reduzieren kann, und argumentiert, dass das Konzentrationslager das politische Paradigma der Moderne sei. ${ }^{44}$ Negri und Hardt sind dagegen optimistisch,

\footnotetext{
${ }^{36}$ Paul B. Preciado: Testo Junkie. Übersetzt von Stephan Geene. Berlin 2016 [zuerst span. 2008].

${ }^{37}$ Ebd., S. 151-232.

${ }^{38}$ Dean: Mediated (wie Anm. 25), S. 237.

${ }^{39}$ Preciado (wie Anm. 36), S. 31.

${ }^{40}$ Ebd., S. 300f.

${ }^{41}$ Dean: Mediated (wie Anm. 25), S. 239.

${ }^{42}$ Vgl. ebd., S. 239-241.

${ }^{43}$ Für die Konstruktion von „Homosexualität“ durch den medizinischen Diskurs siehe David M. Halperin: One Hundred Years of Homosexuality. And Other Essays on Greek Love. New York/London 1990.

${ }^{44} \mathrm{Vgl}$. Giorgio Agamben: Homo sacer. Die souveräne Macht und das nackte Leben. Übersetzt von Hubert Thüring. Frankfurt a. M. 2002 [zuerst ital. 1995].
} 
dass die „Multitude“, eine vielfältige Widerstandsbewegung oder Kraft ohne zentrale Kontrolle, der biopolitischen Ausbeutung im Spätkapitalismus entgegenwirken kann. ${ }^{45}$ Esposito wiederum bietet eine differenziertere Darstellung der Biopolitik als Immunisierung an, die sowohl deren repressive als auch ihre positive Seite erklären kann. ${ }^{46}$

HIV wurde nicht zu den typischen Themen dieser großen Erzählungen über die moderne Biopolitik gezählt. Dennoch verbindet Jaakko Ailio das biopolitische Denken von Roberto Esposito mit einer Untersuchung von HIV. ${ }^{47}$ Dabei zielt er darauf ab, ein Konzept der ,liberalen Thanatopolitik“ zu entwickeln, das Espositos Konzept der nationalsozialistischen Thanatopolitik folgt. Espositos Erklärung der Biopolitik bezieht sich auf das Konzept der Immunität, das heißt einer zerstörerischen Kraft (wie das Immunsystem), die darauf abzielt, Leben oder soziale Institutionen zu schützen. Die Immunität ist jedoch eine Frage des Maßes; wenn zu viel von der schützenden Kraft vorhanden ist, zerstört sie das, was sie schützen soll. Esposito folgend lokalisiert Ailio ein „tödliches Potenzial“48, d. h. die Möglichkeit, dass die Immunität im Liberalismus und seinem subjektiven Menschenrechtssystem zu einer Thanatopolitik wird. Diese Menschenrechte basieren auf dem juristischen Begriff der Person, der vom Körper entkoppelt ist. Ailio argumentiert, dass nur Subjekte als Personen gelten, die eine autonome Kontrolle über ihren Körper haben, was bedeutet, dass nicht alle Menschen als Personen gelten (ausgeschlossen sind z.B. Wahnsinnige ${ }^{49}$ historisch gesehen Schwarze und zeitgenössisch Flüchtlinge). Einige Menschen auf bloße Dinge zu reduzieren, sei daher dem Konzept der

\footnotetext{
${ }^{45}$ Vgl. Michael Hardt/Antonio Negri: Empire. Die neue Weltordnung. Übersetzt von Thomas Atzert und Andreas Wirthensohn. Frankfurt a. M. 2002 [zuerst eng. 2000]; diess: Multitude. Krieg und Demokratie im Empire. Übersetzt von Thomas Atzert und Andreas Wirthensohn. Frankfurt a. M. 2004 [zuerst eng. 2004]; Marianne Pieper/Thomas Atzert/Serhat Karakayalı/Vassilis Tsianos: Empire und die biopolitische Wende. In: Marianne Pieper (Hg.), Empire und die biopolitische Wende. Die internationale Diskussion im Anschluss an Hardt und Negri. Frankfurt a. M. 2007, S. 293-310.

${ }^{46}$ Vgl. Roberto Esposito: Vom Unpolitischen zur Biopolitik. Übersetzt von Francesca Raimondi [zuerst it. 2010]. In: Thomas Bedorf/Kurt Röttgers (Hg.): Das Politische und die Politik. Berlin 2010, S. 89-101; ders.: Immunitas. The Protection and Negation of Life. Übersetzt von Zakiya Hanafi. Cambridge 2011 [zuerst ital. 2002]; ders.: Terms of the Political. Community, Immunity, Biopolitics. Übersetzt von Rhiannon Noel Welch. New York 2013 [zuerst ital. 2008]; Peter Langford: Roberto Esposito. Law, Community and the Political. Abingdon 2015.

${ }^{47}$ Vgl. Jaakko Ailio: Liberal Thanatopolitics and the HIV/AIDS Pandemic. In: Alternatives: Global, Local, Political 38 (2013), H. 3, S. 256-267; ders.: Biopolitics of the Global Governance of HIV/AIDS. In: Sergej Prozorov/Simona Rentea (Hg.): The Routledge Handbook of Biopolitics. London 2017, S. 235-246; ders.: Theory of Biopolitics and the Global Response to HIV/AIDS. From critique to affirmation. Tampere 2017. Siehe auch Niels van Doorn: Treatment is Prevention. In: Cultural Studies 27 (2013), H. 6, S. 901-932, der seine Kritik an PrEP auf Agamben, Negri und Esposito aufbaut. Während sein Argument, dass kommunale Strukturen in Strategien zur Prävention der öffentlichen Gesundheit einbezogen werden sollten, plausibel ist, erklärt van Doorn nicht, warum PrEP ein Problem sei und wie dies mit der Biopolitik verbunden sei.

${ }^{48}$ Ailio: Liberal (wie Anm. 47), S. 261.

${ }^{49}$ Vgl. Michel Foucault: Wahnsinn und Gesellschaft. Eine Geschichte des Wahns im Zeitalter der Vernunft. Übersetzt von Ulrich Köppen. Frankfurt a. M. 1973 [zuerst frz. 1961].
} 
Person und damit auch den liberalen Menschenrechten inhärent. ${ }^{50}$ Bezüglich der liberalen Reaktionen auf HIV, die auf eine wirksame Prävention und Behandlung abzielen, argumentiert Ailio, dass die ungleiche Verteilung des Zugangs zur Behandlung oder Prävention ein Fall liberaler Thanatopolitik sei, der sich jedoch nicht auf einen Ausnahmezustand stütze ${ }^{51}$, sondern aus der Logik der Person folge. Seine Argumentation betont, dass zwei Gruppen von Menschen von der liberalen Form der Person und damit der effizienten HIV-Behandlung ausgeschlossen sind: Menschen in Ländern ohne voll funktionsfähige öffentliche Gesundheitssysteme, die nicht von hilfsfinanzierten HIV-Programmen versorgt werden, sowie undokumentierte Migrant_innen im Westen ohne Zugang zu Behandlung und Präventionsdienstleistungen. ${ }^{52}$

PrEP wird von Ailio nur einmal erwähnt, und zwar als Beleg für die Abschwächung der ersten konservativen Reaktionen auf HIV, die auf Sexnegativität und Stigmatisierung beruhten, und die Verbreitung des rechtebasierten Liberalismus auch in der HIV-Präventionspolitik. ${ }^{53}$ Mit Ailio könnte man argumentieren, dass subjektive Rechte nicht das geeignete politische Mittel sind, um den $\mathrm{Zu}-$ gang zu PrEP zu erweitern. Stattdessen könnte mit Hilfe von Espositos eher vagem Begriff der „Community“54 eine Erweiterung des Zugangs zur PrEP gefordert werden, was man als eine Gesundheitspolitik verstehen könnte, die nicht durch Rechte und Staatsbürgerschaft organisiert wird, sondern für alle offen ist. Eine solche Lösung wäre jedoch ohne den Überbau der biopolitischen großen Erzählungen der italienischen Schule möglich gewesen.

Differenzierte Biopolitik. Rose und Rabinow stehen für einen nuancierteren Ansatz der Biopolitik, der von Foucault und seinem Konzept der (Bio-)Macht ${ }^{55}$ geprägt ist. Im Gegensatz zu Dean und Ailio erklären diese Autoren sowohl die negativen als auch die positiven Seiten der Biopolitik, indem sie die Fähigkeit der ,biological citizens“ hervorheben, ethisch-politische Entscheidungen über biopolitische Fragen zu treffen. ${ }^{56}$ Laut Rose führt die biomedizinische Innovation weder zu einer

\footnotetext{
${ }^{50}$ Vgl. Ailio: Liberal (wie Anm. 47), $260 \mathrm{f}$.

${ }^{51} \mathrm{Vgl}$. Agamben (wie Anm. 44).

${ }^{52}$ Vgl. Ailio: Liberal (wie Anm. 47), S. 261-264.

${ }^{53}$ Vgl. ebd., S. 261.

${ }^{54}$ Ebd., S. 265.

${ }^{55}$ Vgl. Paul Rabinow: French DNA. Trouble in Purgatory. Chicago 1999; ders.: Artificiality and Enlightenment: From Sociobiology to Biosociality. In: Jonathan Xavier Inda (Hg.): Anthropologies of Modernity. Foucault, Governmentality, and Life Politics. Malden 2005, S. 181-193; Nikolas Rose: Molecular Biopolitics, Somatic Ethics and the Spirit of Biocapital. In: Social Theory \& Health 5 (2007), H. 1, S. 3-29; Nikolas Rose/Paul Rabinow: Biopower Today. In: Cisney/ Morar (Hg., wie Anm. 34), S. 297-325.

${ }^{56}$ Vgl. Nikolas Rose: The Politics of Life Itself. Biomedicine, Power, and Subjectivity in the Twenty-First Century.
} 
utopischen Zukunft noch zu einer überwältigend repressiven Pharmamacht, sondern zu einerVielzahl von kleinen Anpassungen, die unserVerständnis unseres Körpers und unseres Lebens erheblich verändern und die in ihrer Gesamtheit Gegenstand biopolitischer Kämpfe sind. Was in derVergangenheit als ,natürlich“ galt, wird zum Gegenstand möglicher Interventionen, die die Gegensätze Natur vs. Kultur, normal vs. pathologisch und Behandlung von Krankheiten vs. Verbesserung der Fähigkeiten verändern und so neue Möglichkeiten und die Notwendigkeit einer politischen Debatte über den Wert verschiedener Möglichkeiten, das biologische Leben zu verändern, zu schaffen: ${ }^{57}$ „Unser biologisches Leben selbst ist nun eine Frage der Entscheidung und Wahl; biopolitische Urteile $\mathrm{zu}$ treffen ist unausweichlich geworden. Das ist es, was es bedeutet, in einem Zeitalter der biologischen Staatsbürgerschaft, der ,somatischen Ethik“ und der ,Vitalpolitik“ zu leben.“58 Diese somatische Ethik ist für Rose eng mit dem Biokapital verbunden. Biomedizinische Interventionen sind zum einen offen für die Kapitalisierung durch Pharmaunternehmen, die eine ethische Genehmigung durch professionelle bioethische Expert_innen benötigen, oft Philosoph_innen, die auf Zuschüsse und Forschungsgelder angewiesen sind. Auf der anderen Seite erfordern biopolitische Kämpfe, dass sowohl Akteure in der Pharmaindustrie als auch Patient_innen und Aktivist_innen ethisch über ihre Entscheidungen und alltäglichen Handlungen in Bezug auf verschiedene biomedizinische Erkenntnisse und Aussagen von Expert_innen nachdenken. Als Ergebnis werden sie auf der Grundlage dieser Technologien neue normative Erwartungen aufbauen und selbst zu Expert_innen werden..$^{59}$

Der HIV/AIDS-Aktivismus ist ein Beispiel für eine solche „biological citizenship“ und veranschaulicht, was Rose „Biosozialität“ nennt. AIDS-Patient_innen und -Aktivist_innen kamen zusammen, um zahlreiche Aufgaben zu erfüllen, wie z.B. Informationen zu verbreiten, Kampagnen für Rechte in Bezug auf Behandlung und Lebensqualität zu organisieren, die gesellschaftliche Stigmatisierung zu bekämpfen und Mitsprache bei der Entwicklung medizinischer Expertise zu fordern. ${ }^{60} \mathrm{Zu} \mathrm{Be}-$ ginn war die Beziehung zwischen der aktivistischen und der traditionell medizinischen Community

Princeton/Oxford 2007, S. 259; Didier Fassin: Another Politics of Life is Possible. In: Theory, Culture \& Society 26 (2009), H. 5, S. 44-60.

${ }^{57}$ Vgl. Rose: Politics (wie Anm. 56), 253 f.

${ }^{58}$ Ebd., S. 254.

${ }^{59}$ Vgl. ebd., S. 257. Siehe für Patient_innenaktivismus Steven Epstein: The politics of health mobilization in the United States: The promise and pitfalls of „,disease constituencies“. In: Social Science \& Medicine 165 (2016), S. 246254; Carlos Novas: Patient Activism and Biopolitis. In: Cisney/Morar (Hg., wie Anm. 34), S. $184-198$.

${ }^{60}$ Vgl. Rose: Politics (wie Anm. 56), S. 144; Steven Epstein: Impure Science. AIDS, Activism, and the Politics of Knowledge. Berkeley 2009. 
antagonistisch, doch bildete sich bald eine Allianz: Auf diese Weise konnten die Mediziner_innen ihre Zielgruppe schwuler Männer erreichen, und die Aktivist_innen wurden zu entscheidenden Akteuren bei der Weiterentwicklung der medizinischen Expertise und der Safer-Sex-Beratung.

Die Konzepte Biokapital, Biosozialität, „biological citizenship“ und somatische Ethik sind gute Werkzeuge, um die Debatten um PrEP zu beschreiben. Das Konzept des Biokapitals bezeichnet die kapitalistische Logik von Big Pharma und die Politik hinter Preisen und Patenten, die den Interessen von Patient_innengemeinschaften und öffentlichen Gesundheitsdienstleistern entgegenwirken. Das Konzept der Biosozialität bezieht sich auf die Tatsache, dass sich eine Gemeinschaft von (potenziellen) PrEP-Nutzern durch ihr Infektionsrisiko konstituiert. „Biological citizenship“ ist der Akt der aktiven Inanspruchnahme und Einforderung von Rechten und der Stärkung einer politikgestaltenden Community. Tatsächlich waren die hauptsächlichen treibenden Kräfte für die Entwicklung von PrEP das öffentliche Gesundheitswesen und die schwule Gemeinschaft und nicht die großen Pharmaunternehmen. ${ }^{61}$ Die somatische Ethik bezieht sich auf die ethischen Praktiken rund um PrEP. Da PrEP bestimmte Praktiken ermöglicht, insbesondere kondomlosen Sex, die oft moralisch sanktioniert werden, stellt sie einen konkreten Fall dar, in dem die somatische Ethik stark umstritten ist. Tatsächlich ergibt sich die Biosozialität von PrEP nicht nur aus biologischen Merkmalen oder Krankheit (wie im klassischen Patient_innenaktivismus) oder dem Risiko von Krankheiten, die mit der Praxis des schwulen Sex generell verbunden sind (wie im klassischen HIV-Aktivismus), sondern es handelt sich dabei um eine spezifische Untergruppe von Schwulen, die sich an ,hochriskanten“ Sexualpraktiken beteiligen, und dieses Engagement wird oft als ethische Wahl konzeptualisiert. Gleichzeitig verändert PrEP die Auffassung dessen, welcher Sex als ,hochriskant' gilt; die biopolitische Technologie wirkt also auf die somatische Ethik.

\section{Die politische Debatte um PrEP}

Während PrEP mittlerweile auch in Deutschland von den Krankenkassen übernommen wird und die Debatte damit zu einem Ergebnis gekommen ist und sich deutlich abschwächt, ist ihre Analyse

\footnotetext{
${ }^{61}$ Die ersten großen PrEP-Studien wurden von öffentlichen Gesundheitsinstitutionen und nicht von Pharmaunternehmen finanziert, siehe Anm. 4 und Daniel Summers: The Battle for Truvada, 2018, https://slate.com/human-interest/2018/05/act-up-is-challenging-gilead-to-make-truvada-more-accessible.html?via=gdpr-consent\&via=gdprconsent [letzter Zugriff am 10.1.2020].
} 
doppelt aktuell: einerseits weil die strukturelle Homophobie und Homonormativität, die darin zu finden ist, weiter wirkt und die Auffassungen zur PrEP prägt, und andererseits, weil dies zu grundsätzlichen Erkenntnissen zum Verhältnis von Bio-Technologie, Sexualität, Ethik und Politik führt, die für eine progressive schwule und queere Politik unerlässlich sind.

Ich schlage vor, vier Positionen schematisch zu unterscheiden, um die biopolitische Debatte um PrEP zu analysieren. Dazu werde ich die Positionen rekonstruieren, die in diesem Bereich zu finden sind, und die Beziehungen zwischen ihnen aufzeigen. Die relevanten Akteure sind insbesondere Aktivist_innen, Wissenschaftler_innen, Mediziner_innen und Mitglieder der schwulen Community. Dabei folge ich lose einer Foucault'schen Analyse von Diskursen und Macht, was zu einem Fokus auf Kämpfe und Auseinandersetzungen über Sexualitätsnormen, sexuelle Subjektivierung und Subjektivität sowie Homophobie führt. ${ }^{62}$ Die Darstellung stützt sich außerdem auf die oben referierte biopolitische Literatur und beleuchtet biopolitische Aspekte von PrEP. Dies ermöglicht es, die biopolitischen Ansätze hinsichtlich ihres analytischen und normativen Potenzials zu bewerten und die These, dass PrEP als ,demokratische Biopolitik' interpretiert werden sollte, zu begründen. Ich behaupte nicht, alle Positionen zu PrEP zu erfassen, die artikuliert wurden. Diese schematische Darstellung hat aber den Vorteil, spezifische Konfliktlinien in den Vordergrund zu stellen. Sie betont unter anderem, wie sich die Biopolitik von PrEP mit Homophobie und Heteronormativität auf der einen Seite und dem realen oder potenziellen demokratischen Charakter der politischen Debatten um PrEP auf der anderen Seite überschneidet.

Die vier hier beschriebenen Positionen sind (1) schwul pro-PrEP, (2) schwul anti-PrEP, (3) professionell pro-PrEP und (4) professionell anti-PrEP. Auf der einen Seite steht die schwule Perspektive und die Debatte innerhalb der schwulen Community zwischen dem Pro-PrEP- und dem AntiPrEP-Lager. Auf der anderen Seite gibt es die nicht-schwule Perspektive von professionellen Mediziner_innen und der Öffentlichkeit. Auch hier findet sich eine Pro-PrEP- und eine Anti-PrEPPerspektive. Einige dieser Perspektiven beziehen sich auf spezifische theoretische Ansätze zur Biopolitik, wie sie oben rekonstruiert wurden. Einige theoretische Elemente der Gouvernementalitätsperspektive finden sich beispielsweise in der schwulen Kritik von PrEP, zum Beispiel, dass Pharmamacht Schwule unter Druck setze, das Medikament zu benutzen. Pro-PrEP-Schwule berufen sich

\footnotetext{
${ }^{62}$ Vgl. Foucault: Wille (wie Anm. 13).
} 
dagegen auf Argumente über die schwule Subjektivität, die in der Debatte über Biopolitik nicht vertreten sind. Die politische Aktivität von schwulen PrEP-Aktivisten als biopolitischen Bürgern passt gut zu Roses und Rabinows biopolitischer Perspektive. Die medizinische pro-PrEP-Position legt den Schwerpunkt auf die Wirksamkeit in Bezug auf Präventionsraten, finanzielle Aspekte und Lebensqualität. Vereinbar mit diesem Argument ist die Kritik an der Ungleichheit des Zugangs zu HIV-Medikamenten durch die Formulierung von Rechten, die das Ergebnis der großen Erzählungen der italienischen Theorie über Biopolitik und PrEP ist. Einige anti-PrEP-Argumente von Mediziner_innen ähneln der Pharmaskepsis von Gouvernementalitätsstudien und basieren, zumindest in Deutschland, auf einer Abneigung gegen die Medikation gesunder Körper, die auf einer naturalistischen Haltung beruht. Homophobie und Homonormativität, ${ }^{63}$ die von biopolitischen Theorien nicht berücksichtigt werden, spielen eine entscheidende Rolle in der Debatte. Dennoch kann der damit verbundene Streit über gute oder schlechte Art und Weisen, schwul zu sein und schwulen Sex zu haben, als eine Auseinandersetzung über somatische Ethik verstanden werden, die an Roses Arbeit anschließend beschrieben werden kann.

Die schwule pro-PrEP Perspektive hat zwei Seiten. Einerseits werden viele Schwule über die medizinischen und gesundheitlichen Vorteile von PrEP informiert und nutzen diese Informationen, um für PrEP zu argumentieren. Ich werde auf diese Argumente näher eingehen, wenn ich die nichtschwule medizinisch-professionelle Pro-PrEP-Position beschreibe. ${ }^{64}$ Auf der anderen Seite gibt es eine nicht-medizinische Argumentation für PrEP, die sich auf die Bedeutung von PrEP für schwule Subjektivität und Erfahrung stützt. ${ }^{65}$ Ich werde diese Argumentation zuerst rekonstruieren, die in Roses Kategorie der somatischen Ethik fällt. Genauer gesagt schlage ich vor, das, worum es hier

\footnotetext{
${ }^{63}$ Das Konzept der Homonormativität beschreibt und kritisiert die konservative Normativität der MainstreamSchwulenpolitik, die sich auf monogame Beziehungen, Ehe und heimischen Konsum konzentriert. Homonormativität bekämpft im Gegensatz zur queeren Kritik die Heteronormativität nicht, sondern stabilisiert sie. Unter Heteronormativität ist dabei der Glaube zu verstehen, dass Heterosexualität eine natürliche Norm ist, sowie die Unterstützung von Institutionen der Heterosexualität, des Sexismus und des Patriarchats, wie zum Beispiel der Ehe. Homonormativität stellt auch einen Unterschied zwischen ,guten' und ,schlechten' Schwulen dar und führt dadurch zu neuen Formen der (internalisierten) Homophobie, siehe Lisa Duggan: The New Homonormativity. In: Russ Castronovo/Dana D. Nelson (Hg.): Materializing Democracy. Towards a Revitalized Cultural Politics. Durham/London 2002, S. 175194; Kevin P. Murphy/Jason Ruiz/David Serlin (Hg.): Queer Futures. Radical History Review 8 (2008), H. 100. ${ }^{64}$ Die Unterscheidung zwischen der schwulen und der nicht-schwulen medizinischen Perspektive ist nur bis zu einem gewissen Grad möglich, da viele HIV-Mediziner_innen schwul sind und die HIV-Forschung teilweise aus dem HIV-Aktivismus der schwulen Community entwickelt wurde.

${ }^{65}$ Vgl. Judith D. Auerbach/Trevor A. Hoppe: Beyond ,getting drugs into bodies“: social science perspectives on preexposure prophylaxis for HIV. In: Journal of the International AIDS Society 18 (2015), H. 4, Suppl. $3,19983$.
} 
geht, sexuell-somatische Ethik zu nennen: Die Aushandlung von Politik, Subjektivität, sexuellem Vergnügen und Begehren, sexuellen Normen und medizinischen Technologien. Der Ausgangspunkt hierfür ist, dass Sex ohne Kondome von vielen als besser empfunden wird als Sex mit Kondomen. Doch selbst eine Äußerung von dieser Banalität ist in einem Klima moralisierter Sexualität gefährlich. Es handelt sich um eine Aussage, die nicht in einen Diskurs von Vernunft und Verantwortung fällt, sondern ,nur den Bereich von Begehren und Lust betrifft. Die unmittelbare Reaktion der meisten Menschen auf diese Aussage ist, dass dies unverantwortlich sei angesichts der Gefahren des kondomlosen Sex und des relativ geringen Aufwandes, der mit derVerwendung eines Kondoms verbunden ist. Zusätzlich trägt zur moralischen Verurteilung die Auffassung bei, dass das Risiko, andere mit STIs zu infizieren, bei kondomlosem Sex erhöht sei. Begehren und Lust sind keine starken Argumente in diesem Diskurs über Verantwortung, Vernunft und Schuld. Prävention bedeutet in diesem Paradigma ausschließlich, die Menschen über bestimmte Risiken zu informieren, in der Annahme, dass sie dadurch beim Sex ,rationale‘ Entscheidungen treffen (z.B. ein Kondom zu verwenden). ${ }^{66}$

Dieser Widerstand gegen den Wunsch nach kondomlosem Sex zeigt etwas zutiefst Problematisches im aktuellen Zustand der schwulen Sexualität und Subjektivierung - und PrEP wird von vielen Schwulen als Antwort auf dieses grundlegendere Problem angesehen. Offensichtlich ist die eklatante und offene Homophobie ein großes Problem, die sich in den letzten Jahren im Westen sogar noch verstärkt hat, weil Rechtsbewegungen entstanden sind, die homophobe Hassreden als legitime Positionen im öffentlichen Diskurs darstellen. ${ }^{67}$ Doch selbst innerhalb des diversitätsaffirmativen Libe-

\footnotetext{
${ }^{66}$ Für eine Kritik an diesem rationalistischen Paradigma, das schwule Erfahrung, Subjektivität, Fantasie, Sex und Begehren auslässt, siehe Dean: Unlimited (wie Anm. 34); Barry D. Adam: Epistemic fault lines in biomedical and social approaches to HIV prevention. In: Journal of the International AIDS Society 14 (2011), Suppl. 2, S. 1-9; Kane Race: Framing Responsibility. HIV, Biomedical Prevention, and the Performativity of the Law. In: Journal of Bioethical Inquiry 9 (2012), H. 3, S. 327-338; Tim Dean: The Biopolitics of Pleasure. In: South Atlantic Quarterly 111 (2012), H. 3, S. 477-496; ders.: Mediated (wie Anm. 25); ders.: No Sex Please, We're American. In: American Literary History 27 (2015), H. 3, S. 614-624; Halperin: Biopolitics (wie Anm. 34); David M. Halperin: What is Sex for? In: Critical Inquiry 43 (2016), H. 1, S. 1-31; Mathieu Trachman/Gabriel Girard: Targeting Fallible Men. Communication Strategies and Moral Issues in a Pre-exposure Prophylaxis Trial. In: Journal of Acquired Immune Deficiency Syndromes 79 (2018), Suppl. 1, S. S13-S19.

${ }^{67}$ In Deutschland besteht beispielsweise ein Zusammenhang zwischen der wachsenden politischen Macht der homophoben und rechtspopulistischen Partei Alternative für Deutschland (AfD), dem Aufkommen homophober Einstellungen und dem Aufkommen homophober Hassverbrechen, die viele Akteure als Kausalität interpretieren, vgl. LSVD: AfD - eine unberechenbare Alternative, https://www.lsvd.de/politik/rechtspopulismus-entgegentreten/afdeine-unberechenbare-alternative.html [letzter Zugriff am 2.1.2020]; Oliver Decker/Johannes Kiess (Hg.): Die enthemmte Mitte. Autoritäre und rechtsextreme Einstellung in Deutschland. Die Leipziger Mitte-Studie 2016. Gießen
} 
ralismus, wo Homophobie zu fehlen scheint und Homosexuelle glücklich verheiratet sind, strukturiert Homophobie die schwule Subjektivität und Sexualität grundlegend. Es ist jetzt größtenteils akzeptiert, schwul zu sein, aber nur, wenn man ein ,guter Schwuler` ist. Wenn man ein normalisiertes, bürgerliches und erfolgreiches Leben führt, ein Leben der Homonormativität, das heteronormativen Regeln folgt, spielt das Schwulsein keine Rolle. Diese Akzeptanz des bürgerlichen Schwulseins ist ein Erfolg der Schwulenbewegung, der durch die Bekämpfung der Stereotypen von hypersexualisierten und verweichlichten Schwulen erreicht wurde, indem sich diese Schwulen als ,normal', männlich und desexualisiert präsentierten und benahmen - das ist Homonormativität. MainstreamSchwule präsentieren ihre sexuelle Orientierung als zufällige, nicht wesentliche Eigenschaft ihrer Persönlichkeit; sie bestimme nicht, wer sie sind. Mit der Gleichstellung in der Ehe erreichte diese Bewegung zur Normalisierung ihren Höhepunkt, und viele Homosexuelle verhalten sich heutzutage genau wie Heteros und freuen sich über soziale Anerkennung und Akzeptanz. Aber diese Akzeptanz geht auf Kosten einer neuen Exklusion. Trans ${ }^{\star}$ - und gender-non-conforming Menschen, queers of color und schwule Männer, die anderen Sex haben als in einer langfristigen romantischen Beziehung, sind von dieser Homonormativität ausgeschlossen. ${ }^{68}$

Während der schwule Stolz der ,guten Schwulen` die Oberfläche des zeitgenössischen Liberalismus

2016, S. 51; Clara Beiker: Hate crimes against homosexuals on the rise in Germany, 2017, https://www.dw.com/en/hate-crimes-against-homosexuals-on-the-rise-in-germany/a-40028141 [letzter Zugriff am 3.1.2020]; LSVD: Ergebnisse des ersten Vernetzungstreffen, 21. Oktober 2017. Leipzig 2017. Eine soziale und politische Bewegung gegen Geschlechtergleichstellung, sexuelle Emanzipation und Selbstbestimmung mit vielen personellen und thematischen Überschneidungen mit rechten Bewegungen und Parteien agierte in Europa in den letzten Jahren offener und wurde mächtiger, vgl. Sabine Hark/Paula-Irene Villa (Hg.): Anti-Genderismus. Sexualität und Geschlecht als Schauplätze aktueller politischer Auseinandersetzungen. Bielefeld 2015; Roman Kuhar/David Paternotte (Hg.): Anti-Gender Campaigns in Europe. Mobilizing Against Equality. London 2017. Einige westeuropäische rechte Parteien definieren Toleranz für verschiedene Lebensstile als ,europäisch', um Muslime, Islam und People of Color als homophob und unzivilisiert abzulehnen. Es ist wichtig zu beachten, dass diese scheinbar schwulenfreundliche Rhetorik nur instrumentell zur Förderung des anti-muslimischen Rassismus eingesetzt wird, vgl. Scott Siegel: Friend or Foe? The LGBT Community in the Eyes of Right-Wing Populism. In: Europe Now, 2017, https://www.europenowjournal.org/2017/07/05/friend-or-foe-the-lgbt-community-in-the-eyes-of-right-wing-populism/ [letzter Zugriff am 3.1.2020]. Queere Organisationen kämpfen gegen die Instrumentalisierung ihrer Anliegen für rassistische Projekte und weisen darauf hin, dass Homo- und Transphobie ein allgemeines Problem in der Gesellschaft ist. Diese seien nicht allein bei Muslim_innen zu finden, sondern werden durch (rechte) antiegalitäre Ideologien gefördert.

Siehe zum Beispiel GLADT: Redebeitrag von GLADT bei der „Demonstration gegen antimuslimischen Rassismus“, 2009, https://www.yumpu.com/de/document/view/21404779/demonstration-gegen-antimuslimischen-rassismusgladt [letzter Zugriff am 3.1.2020].

${ }^{68}$ Vgl. Jayson Flores: Everything You Need to Know About Homonormativity, 2017, https://www.pride.com/firstperson/2017/10/12/what-homonormativity [letzter Zugriff am 3.1.2020]. Vgl. zur Beziehung von Homonormativität und Biopolitik Mike Laufenberg: Sexuelle Immunologik. Heteronormativität als biopolitischer Sicherheitsmechanismus. In: María Teresa Herrera Vivar/Petra Rostock/Uta Schirmer/Karen Wagels (Hg.): Über Heteronormativität. Auseinandersetzungen um gesellschaftliche Verhältnisse und konzeptuelle Zugänge (Forum Frauen- und Geschlechterforschung, Bd. 45). Münster 2016, S. 51-69. 
ist, ist die schwule Schande der ,schlechten Schwulen“ ihre Kehrseite. ${ }^{69}$ Dies schafft eine Konstellation aus Scham und Schuld um schwulen Sex. ${ }^{70}$ Die AIDS- und die Post-AIDS-Generationen wuchsen mit einer tiefen Angst vor schwulem Sex auf. ${ }^{71}$ Sex war nicht nur etwas Schändliches, sondern auch etwas Gefährliches. Sich daran zu beteiligen war schon problematisch genug, aber es auf eine ,unvernünftige' und leichtfertige Weise zu tun und sich mit HIV oder anderen STIs zu infizieren, drängte Schwule aus dem Rahmen der liberalen Akzeptanz von Homosexualität heraus. Während Schuld nicht mehr für das Schwulsein allein zugewiesen wird, wird sie umso mehr für die Ausübung von nicht-normativem und, unverantwortlichem' Sex zugewiesen. Daher konstituiert sich der schwule Sex um eine Ökonomie der Schuld, die sich aus der liberalen und homonormativen Verfeinerung der Homophobie ergibt, die sich zu einem wesentlichen Teil auf Kondome stützt. ${ }^{72}$ Das Kondom ist das perfekte Schuldwerkzeug, und viele Schwule berichten von psychischen Selbstquälereien, nachdem sie vergessen haben, Kondome zu benutzen, nicht nur wegen der Angst vor einer Infektion, sondern auch wegen des Stigmas, das mit dem vermeintlich unverantwortlichen Verhalten zusammenhängt, dem eine Infektion zugeschrieben werden würde. Unter dieser Rubrik ist schwuler Sex auf Kondom-Basis untrennbar mit Schuld, Angst und verinnerlichter Homophobie verbunden. PrEP ist ein neues Kapitel im queeren Kampf gegen Homophobie und hilft schließlich, schwulen Sex aus seinem Zusammenhang mit Krankheit und Tod zu lösen, den er seit etwa 40 Jahren hatte. ${ }^{73}$

\footnotetext{
${ }^{69}$ Vgl. David M. Halperin/Valerie Traub (Hg.): Gay Shame. Chicago 2009.

${ }^{70}$ Vgl. Amy L. Hequembourg/Ronda L. Dearing: Exploring Shame, Guilt, and Risky Substance Use Among Sexual Minority Men and Women. In: Journal of Homosexuality 60 (2013), H. 4, S. 615-638.

${ }^{71}$ Vgl. Michael P.: „Without the constant fear of HIV infection, I can engage in sex with the love of my life“, 2015, http://myprepexperience.blogspot.com/2015/05/michael-without-constant-fear-of-hiv.html [letzter Zugriff am 3.1.2020]; Matt Cain: Sex without fear - my experiment with the HIV-prevention drug PrEP. In: The Guardian vom 22.6. 2017, https://www.theguardian.com/society/2017/jun/22/sex-without-fear-my-experiment-with-hivpreventative-drug-prep [letzter Zugriff am 3.1.2020].

${ }^{72}$ Kane Race: Reluctant Objects. In: GLQ: A Journal of Lesbian and Gay Studies, 22 (2016), H. 1, S. 1-31, beschreibt die Anti-PrEP-Einstellungen in der Schwulen-Community als Angst vor Sex.

${ }^{73}$ Vgl. Shane P. Collins/ Vanessa M. McMahan/Joanne D. Stekler: The Impact of HIV Pre-exposure Prophylaxis (PrEP) Use on the Sexual Health of Men Who Have Sex with Men: A Qualitative Study in Seattle, WA. In: International Journal of Sexual Health 29 (2017), H. 1, S. 55-68; Kimberly Koester/Rivet K. Amico/Hailey Gilmore/Albert Liu/Vanessa McMahan/Kenneth Mayer/Sybill Hosek/Robert Grant: Risk, safety and sex among male PrEP users: time for a new understanding. In: Culture, Health \& Sexuality 19 (2017), H. 12, S. 1301-1313; Sky Gilbert: Gay men: Finally, sex without fear, 2018, http://theconversation.com/gay-men-finally-sex-without-fear-100638 [letzter Zugriff am 14.3.2018]; Daniel Grace/Jody Jollimore/Paul MacPherson/Matthew J.P. Strang/Darrell H.S. Tan: The Pre-Exposure Prophylaxis-Stigma Paradox: Learning from Canada's First Wave of PrEP Users. In: AIDS Patient Care and STDs 32 (2018), H. 1, S. 24-30. Exemplarisch für diese befreiende Funktion von PrEP für Schwule in Bezug auf Schuld und Kondome ist ein öffentlicher Beitrag auf Facebook, der vielfach geteilt wurde, vgl. Christoph Hartmann: [PrEP-Post], https://www.facebook.com/ophtopic/posts/10213252029390502 [letzter Zugriff am 14.8.2018].
} 
Der befreiende Aspekt von PrEP betrifft nicht nur Homophobie und soziale Stigmatisierung, sondern verändert und entlastet auch die schwule Sexualität: PrEP reduziert die Notwendigkeit einer ständigen Aushandlung des Schutzes vor Krankheiten beim schwulen Sex. Um verantwortungsbewusst zu handeln und im sexuellen Paradigma von Kondomen keine Schuld auf sich zu laden, müssen Schwule ständig die Risiken des Sexes reflektieren und verhandeln. ${ }^{74}$ Sie müssen Annahmen darüber treffen, wie ,gefährlich“ der Partner ist und ob sie ihm vertrauen können. Vor allem der passive Partner beim Analverkehr hat kaum Kontrolle über die Verwendung von Kondomen und muss manchmal während des Geschlechtsverkehrs manuell überprüfen, ob das Kondom noch vorhanden ist. ${ }^{75}$ So ist Sex für viele Schwule konstant mit Sorgen und Angst verbunden. PrEP kann diese Bedingungen ändern. Dies bedeutet, dass man erstmals selbst effizient Verantwortung übernehmen kann, indem man sie auf die Einhaltung der medikamentösen Behandlung verlagert, weg von der oft unkontrollierbaren Dynamik des Sexes. Hier liegt ein offener Widerspruch zu Deans Position, die diese Verschiebung derVerantwortung nicht als befreiend, sondern als Intensivierung der Rationalisierung und Disziplinierung der Sexualität interpretiert, da zum ersten MalVerantwortung objektiv durch Medikamentenspiegel im Blut gemessen werden kann. ${ }^{76}$

An dieser Erläuterung zeigt sich ein allgemeinerer Punkt: Sexualethik, soziale Normen und (Medizin-) Technologie sind miteinander verflochten und bilden Dispositive der Macht. Aufbauend auf Roses Begriff der somatischen Ethik kann diese Art der ethischen Problematisierung als sexuellsomatische Ethik bezeichnet werden. Es handelt sich um eine Reflexion und Weiterentwicklung von Gemeinschaftsnormen, persönlichen Entscheidungen und Wünschen, die die Medizintechnik aushandelt.

Die Anti-PrEP-Schwulenperspektive umfasst ebenfalls einerseits medizinische Argumente und andererseits Argumente, die sich mit Subjektivität, sexuellen Normen und Politik befassen und die unter die Rubrik der somatischen Ethik fallen. Wie bereits erwähnt, werde ich hier nur die ethischen Argumente rekonstruieren und die medizinischen Argumente, die von Schwulen wie von nicht-

\footnotetext{
${ }^{74}$ Bezüglich der Aushandlung der Verantwortung siehe Ingrid Young/Paul Flowers/Lisa McDaid: Can a pill prevent HIV? Negotiating the biomedicalisation of HIV prevention. In: Sociology of Health \& Illness 38 (2016), H. 3, S. 411-425.

${ }^{75} \mathrm{Vgl}$. Conversation about contemporary politics of PrEP with Emmanuel Danan (19.7.2018).

${ }^{76}$ Vgl. Dean: Mediated (wie Anm. 25), S. 233.
} 
schwulem medizinischem Fachpersonal und der öffentlichen Debatte verwendet werden, diskutieren. Schwule Gegner von PrEP argumentieren, dass sie die schwule Sexualität erheblich verändert und eine Kultur des kondomlosen Sexes fördert, die die Freiheit derjenigen, die Kondome benutzen wollen, einschränkt.Viele Berichte und Beschwerden von Schwulen in westlichen Großstädten, in denen die PrEP besonders verbreitet ist, zeigen, dass es schwieriger wurde, über Apps Treffen zum Sex zu organisieren, wenn man auf Kondomgebrauch besteht. ${ }^{77}$ Sex ist eine kulturelle Praxis und die Teilnehmer werden in eine Sexualkultur subjektiviert. Es gibt keinen wesenhaft guten und natürlichen Sex, sondern dieser wird immer durch Normen und Technologien vermittelt. Vor PrEP war die Verwendung von Kondomen ein Standard und unbestrittene Norm, weswegen sie für viele Schwule als nicht störend akzeptiert wurde, obwohl die Kondom-Benutzung für eine beträchtliche Anzahl von Schwulen ein Problem war. ${ }^{78}$ Die Möglichkeit des vor einer HIV-Infektionen geschützten, kondomlosen Sex verändert diese sexuelle Subjektivierung. Das Kondom wird in Frage gestellt und wird zum Gegenstand einer sexualethischen Auseinandersetzung, bei der viele sich kondomlosen Sex wünschen und andere das Kondom als einziges Mittel für sicheren Sex verteidigen. Die sexuelle Subjektivierung hin zu kondomlosem Sex wird von vielen als Druck gesehen, gegen ihren Willen ebenfalls PrEP einzunehmen. PrEP könnte zur neuen Norm werden, an die man sich halten muss, um an der veränderten Sexualkultur teilhaben zu können.

Anti-PrEP-Argumente von Schwulen stützen sich auch auf Argumente, die mit der biopolitischen Kritik der Gouvernementalitätsanalysen übereinstimmen. Auch wenn sie sich nicht auf Foucault und den Begriff ,Subjektivierung، beziehen, kann die schwule Anti-PrEP-Kritik, die ich rekonstruiert habe, durch dieses Konzept erfasst werden. Einige verbinden die sexuelle Subjektivierung, d. h. die veränderten Gemeinschaftsnormen und den damit verbundenen Druck auf den Einzelnen, mit den Gesundheitsbehörden und der Pharmaindustrie. Das Argument ist, dass PrEP von Pharmaunternehmen gefördert wird, um neue Märkte zu erschließen und PrEP-Nutzer wirtschaftlich auszunutzen, indem die sexuelle Subjektivierung verändert wird. Offensichtlich ist es aus Sicht der Pharmaindustrie begrüßenswert, dass nicht infizierte Menschen Medikamente nehmen, da ihre Zahl die Zahl der Infizierten übersteigt. ${ }^{79}$ In diesem Zusammenhang reiht sich PrEP in den allgemeinen

\footnotetext{
${ }^{77}$ Vgl. Martin Holt/Toby Lea/Limin Mao/Johann Kolstee/Iryna Zablotska/Tim Duck: Community-level changes in condom use and uptake of HIV pre-exposure prophylaxis by gay and bisexual men in Melbourne and Sydney, Australia: results of repeated behavioural surveillance in 2013-17. In: The Lancet HIV 5 (2018), H. 8, S. e448-e456.

${ }^{78}$ Vgl. Halperin: Gay Men (wie Anm. 34), S. 11-37; Dean: Unlimited (wie Anm. 34).

${ }^{79}$ Vgl. Nils Behnke/Michael Retterath/Todd Sangster/Ashish Singh: New Paths to Value Creation in Pharma. In a
} 
Trend der Medikalisierung und insbesondere der Pharmaisierung - also nicht nur der zunehmenden Vergabe von Medikamenten, sondern auch des zunehmenden Einflusses der Pharmaindustrie auf die Vergabepraxis und Gesundheitspolitik ${ }^{80}$ - der Prävention ein. Solche Argumente übersehen jedoch, dass die Entwicklung von PrEP tatsächlich in einer Interaktion zwischen der schwulen und Public Health-Communities gestaltet wurde, indem die schwulen Interessensvertreter frühzeitig in die Prozesse der drei wichtigsten PrEP-MSM-Studien iPrEx, Ipergay und Proud integriert wurden. ${ }^{81}$ Die ersten MSM-PrEP-Studien wurden nicht von der Firma Gilead, die das Patent auf Truvada innehatte, finanziert, die nur die Medikamente und Placebos stiftete, sondern von staatlichen Forschungseinrichtungen. ${ }^{82}$

Neben der Besorgnis über den Wandel der sexuell-somatischen Ethik und über PrEP als Projekt

changing industry, survival increasingly depends on leading in categories and distinctive business capabilities, 2014, https://www.bain.com/insights/new-paths-to-value-creation-in-pharma/ [letzter Zugriff am 14.3.2018]; Elvira Thissen: Focus on pharma: Creating a market for disease prevention, 2014, https://www.greenbiz.com/article/focuspharma-creating-market-disease-prevention [letzter Zugriff am 14.6.2018].

${ }^{80} \mathrm{Vgl}$. Mara Tognetti Bordogna: From Medicalisation to Pharmaceuticalisation - A Sociological Overview. New Scenarios for the Sociology of Health. In: Social Change Review 12 (2014), H. 2, S. 119-140.

${ }^{81}$ Vgl. Gus P. Cairns/Kane Race/Petro Coicochea: PrEP: controversy, agency and ownership. In: Journal of the International AIDS Society 19 (2016), H. 7(Suppl. 6), S. 21120, hier 2. Dennoch gibt es bei einigen PrEP-Studien erhebliche Probleme, die auf den globalen Kapitalismus und Pharmagewinninteressen zurückzuführen sind. Insbesondere die Partners-Studie (vgl. Jared M. Baeten/Deborah Donnell/Patrick Ndase/Nelly R. Mugo/James D. Campbell/Jonathan Wangisi/Jordan W. Tappero/Elizabeth A. Bukusi/Craig R. Cohen/Elly Katabira/Allan Ronald/Elioda Tumwesigye/Edwin Were/Kenneth H. Fife/James Kiarie/Carey Farquhar/Grace John-Stewart/Aloysious Kakia/Josephine Odoyo/Akasiima Mucunguzi/Edith Nakku-Joloba/Rogers Twesigye/Kenneth Ngure/Cosmas Apaka/Harrison Tamooh/Fridah Gabona/Andrew Mujugira/Dana Panteleeff/Katherine K. Thomas/Lara Kidoguchi/Meighan Krows/Jennifer Revall/Susan Morrison/Harald Haugen/Mira Emmanuel-Ogier/Lisa Ondrejcek/Robert W. Coombs/Lisa Frenkel/Craig Hendrix/Namandjé N. Bumpus/David Bangsberg/Jessica E. Haberer/Wendy S. Stevens/Jairam R. Lingappa/Connie Celum: Antiretroviral Prophylaxis for HIV Prevention in Heterosexual Men and Women. In: The New England Journal of Medicine 367 (2012), H. 5, S. 399-410) ist problematisch wegen des Auslagerns einer riskanten Studie in arme Ländern des globalen Südens (hier Uganda und Kenia), um Medikamente zur Behandlung von Patienten im globalen Norden zu entwickeln. Ein weiteres Problem mit der Partners-Studie war, dass sie für HIV-positive Patient_innen vorgesehene Medikamente an HIV-negativen Personen für den Einsatz als PrEP transferierte, vgl. Patton/Kim (wie Anm. 35). Erste PrEP-Studien in Kamerun und Kambodscha in den frühen 2000er Jahren wurden aufgrund von Verstößen gegen ethische Standards gestoppt , da sie Teilnehmer_innen einem Infektionsrisiko aussetzten, nachdem Act Up Paris gegen die Studien protestiert hatte, siehe Jerome A. Singh/Edward J. Mills: The Abandoned Trials of Pre-Exposure Prophylaxis for HIV: What Went Wrong? In: PLoS Medicine 2 (2005), H. 9, e234.

${ }^{82}$ Die iPrEx-Studie wurde hauptsächlich von den U.S. National Institutes of Health (NIH) finanziert (vgl. Grant u. a. [wie Anm. 4]), die Ipergay-Studie hauptsächlich von der French Agency for Research on AIDS and Viral Hepatitis (ARNS, vgl. Molina u. a. [wie Anm. 8]) und die PROUD-Studie hauptsächlich von der British Medical Research Council Clinical Trials Unit am University College London und Public Health England (vgl. McCormack u. a. [wie Anm. 4]). Die derzeit laufende Discover-Studie vergleicht Truvada und Descovy und wird vollständig von Gilead finanziert, vgl. AVAC (wie Anm. 7). Descovy ist eine leicht modifizierte Version von Truvada, die weniger Nebenwirkungen haben soll. Gilead muss die Vorteile von Descovy gegenüber Truvada beweisen, um die Gewinne hoch zu halten, nachdem das Patent von Truvada kürzlich abgelaufen ist, was den Markt für billigere Generika von Truvada öffnete. 
von „Big Pharma‘ zur Ausbeutung von Schwulen gibt es auch eine rundheraus hasserfüllte homophobe Stigmatisierung von PrEP-Nutzern innerhalb der schwulen Gemeinschaft. ${ }^{83}$ Es ist die innergemeinschaftliche Version der homophoben Schuld- und Schamökonomie des Sex, die, wie oben beschrieben, zur homonormativen Konstruktion von guten und schlechten Schwulen führt. Ein Beispiel für dieses PrEP-Shaming ist das Wort „,Truvada Whore“ (Truvada-Schlampe), das als Hassrede gegen Schwule, die PrEP nutzen, verwendet wird und sich in erniedrigender Weise auf ihre angeblich promiskuitive Sexualität bezieht. ${ }^{84}$ In einem Akt der typischen queeren Neuinszenierung wurde dieses Konzept von Pro-PrEP-Schwulen und PrEP-Nutzern schnell wieder angeeignet ${ }^{85}$ und in eine stolze Selbstbezeichnung verwandelt, die Kritik an sozialer Stigmatisierung und Slut Shaming zum Ausdruck bringt. ${ }^{86}$ Die deutlichsten Beispiele für Hassrede gegen PrEP-Nutzer_innen ist auf Online-Hook-Up- und Dating-Netzwerken zu finden. Die folgenden Zitate stammen aus Screenshots von Online-Dating-Gesprächen, die ich vom PrEP-Aktivisten Emmanuel Danan aus Berlin erhalten habe. ${ }^{87}$ Sie zeigen deutlich ein HIV- und PrEP-Stigma in der schwulen Community. Die Beleidigungen werden oft in der Sprache derVerantwortung und auf Basis von Fehlinformationen über die Medizintechnik, ihre Effizienz und vorhandene Risiken formuliert. Sie zeigen, wie wichtig es für einige Schwule ist, auf der ,guten' Seite zu stehen, ein Wunsch, der leider oft dadurch ausgetragen wird, andere wegen ihrer Sexualität und ihrer (gut informierten) Präventionswahl zu stigmatisieren: „You're making the responsible people pay for what the unresponsible people are doing.“ - „Oh look, one of those fags that's proud to be a who're! Lol. Gay pride!!“ - „Go fuck Poz guys you sicko.Your gross as fuck. I keep blocking you but you keep making new profiles. No one cares if your on pRep. Ok“ - „Prep. Fuckin disgusting. Dirty breeder. Prep is to stop HIV only. Not other vile STD“ - „,baresex ist jedenfalls unverantwortlich. wird Zeit daß die AFD Listen anlegt mit Leuten wie dir und sowas eingesperrt wird. Sicherungsverwahrung oder Endlösung. “88

\footnotetext{
${ }^{83}$ Vgl. Sarah K. Calabrese/Kristen Underhill: How Stigma Surrounding the Use of HIV Preexposure Prophylaxis Undermines Prevention and Pleasure. A Call to Destigmatize „Truvada Whores“. In: American Journal of Public Health 105 (2015), H. 10, S. 1960-1964; Grace u. a. (wie Anm. 73).

${ }^{84}$ Vgl. David Duran: Truvada Whores?, 2012, https://www.huffingtonpost.com/david-duran/truvada-whores_b_2113588.html [letzter Zugriff am 9.1.2020].

${ }^{85}$ Vgl. Adam D. Galinsky/Cynthia S. Wang/Jennifer A. Whitson/Eric M. Anicich/Kurt Hugenberg/Galen V. Bodenhausen: The Reappropriation of Stigmatizing Labels. The Reciprocal Relationship Between Power and Self-Labeling. In: Psychological Science 24 (2013), H. 10, S. 2020-2029.

${ }^{86}$ Vgl. David Duran: An Evolved Opinion on Truvada, 2014, https://www.huffingtonpost.com/david-duran/truvadawhore-an-evolved-o_b_5030285.html?guccounter=2 [letzter Zugriff am 9.1.2020]; pupbones: I am a Truvada Whore, 2014, http://myprepexperience.blogspot.com/2014/03/i-am-truvada-whore.html [letzere Zugriff am 9.1.2020].

${ }^{87}$ Vgl. Danan (wie Anm. 75).

${ }^{88}$ Ich habe die Texte der Chats bei der Transkription nicht verändert.
} 
Trotz dieses schweren HIV- und PrEP-Stigmas und der damit verbundenen Hassrede gibt es auch gute Nachrichten: Der vermehrte Einsatz von PrEP führt bereits zu einem messbar geringeren HIVund PrEP-bezogenen Stigma beim schwulen Online-Dating. ${ }^{89}$ Dies unterstützt die These vieler Schwuler, dass PrEP eine befreiende Wirkung auf schwulen Sex haben kann.

Die Pro-PrEP-Stimmen aus dem Gesundheitswesen weisen darauf hin, dass PrEP ein nützliches, effizientes und kostengünstiges Instrument zur Senkung der HIV-Infektionsraten gefährdeter Bevölkerungsgruppen ist und daher eine notwendige Komponente jeder Strategie zur Bekämpfung und endgültigen Beendigung von HIV/AIDS darstellt. Da die Effizienz von PrEP heute unbestritten ist, werde ich mich auf zwei Probleme konzentrieren, die durch PrEP-Kritiker_innen aufgeworfen wurden und die Antworten der PrEP-Unterstützer_innen darauf schildern: die potenzielle Verbreitung anderer Geschlechtskrankheiten aufgrund steigender Raten von kondomlosem Sex und das Problem der Finanzierung.

Ein Argument gegen PrEP ist die Annahme, dass sie zur Ausbreitung von anderen sexuell übertragbaren Infektionen führe, da PrEP eine Kultur des kondomlosen Sex fördere. Dafür gibt es ein paar Belege. ${ }^{90}$ Das Argument von Mediziner_innen und schwulen PrEP-Aktivisten gegen diese Befürchtung ist, dass Kondome erstens nicht gut funktionieren, wenn es darum geht, die anderen STIs (hauptsächlich Gonorrhö, Chlamydien, Syphilis) zu verhindern, so dass der Unterschied in den Infektionsraten nicht signifikant sei. Zweitens helfe PrEP im Gegenteil bei der Bekämpfung dieser anderen STIs, da es viele gefährdete Menschen dazu bringt, sich regelmäßig auf diese STIs testen zu lassen, da das PrEP-Regime eine allgemeine Überprüfung der sexuellen Gesundheit alle drei Monate verlangt. ${ }^{91}$ Das Gesundheitswesen wendet viel Energie auf, um gefährdete Menschen dazu zu

\footnotetext{
${ }^{89}$ Vgl. Sarit A. Golub/Corina Lelutiu-Weinberger/Anthony Surace: Experimental Investigation of Implicit HIV and Preexposure Prophylaxis Stigma. Evidence for Ancillary Benefits of Preexposure Prophylaxis Use. In: Journal of Acquired Immune Deficiency Syndromes 77 (2018), H. 3, S. 264-271.

${ }_{90}$ Vgl. Vinh-Kim Nguyen/Zoë R. Greenwald/Helen Trottier/Martha Cadieux/Alexandre Goyette/Mariève Beauchemin/Louise Charest/Danièle Longpré/Stéphane Lavoie/

Hermione Gbego Tossa/Réjean Thomas: Incidence of sexually transmitted infections before and after preexposure prophylaxis for HIV. In: AIDS 32 (2018), H. 4, S. 523-530.

${ }^{91}$ Vgl. Hyman M. Scott/Jeffrey D. Klausner: Sexually transmitted infections and pre-exposure prophylaxis: challenges and opportunities among men who have sex with men in the US. In: AIDS Research and Therapy 13 (2016), S. 5; Michalina A. Montano/Julia C. Dombrowski/Lindley A. Barbee/Matthew R. Golden/Christine M. Khosropour: Changes in Sexual Behavior and STI Diagnoses Among MSM Initiating PrEP in a Clinic Setting. In: AIDS and Behavior 23 (2019), H. 2, S. 548-555.
} 
bringen, sich testen zu lassen, aber die Öffentlichkeitsarbeit ist schwierig, besonders innerhalb eines rationalistischen Paradigmas der Sexualaufklärung. Mit PrEP kommen Menschen, die einem besonderen Risiko bezüglich STIs ausgesetzt sind (mit oder ohne PrEP), zum Arzt und lassen sich freiwillig testen, um die PrEP-Medikamente zu erhalten. Das zweite Argument gegen PrEP ist, dass sei teuer sei. Mehrere Studien zeigen jedoch, dass es kostengünstig ist, einem erhöhten Risiko ausgesetzten Bevölkerungsgruppen PrEP zugänglich zu machen, da die Kosten für die lebenslange Behandlung einer infizierten Person weitaus höher sind als die Kosten für PrEP. ${ }^{92}$

Das größte Bedenken von medizinischen Fachkräften ist derzeit die ungleiche Verteilung von PrEP auf gefährdete gesellschaftliche Gruppen. Während PrEP in schwulen cis-identifizierten Bevölkerungsgruppen mit privilegiertem sozialem und ökonomischen Status mehr und mehr akzeptiert und begrüßt wird, haben Gruppen, die von unterschiedlichen Diskriminierungsverhältnissen betroffen sind, teilweise einen erschwerten Zugang zu PrEP, obwohl sie in vielen Ländern einem erhöhten HIV-Risiko ausgesetzt sind. Dazu gehören MSM, die sich nicht als schwul identifizieren, transgender Menschen, schwule und heterosexuelle People of Color und Migrant_innen. ${ }^{93}$ Dies liegt zum Teil daran, dass Sexualaufklärungsprogramme auf Rationalität, Risikomanagement und

\footnotetext{
${ }^{92}$ Vgl. Jessie L. Juusola/Margaret L. Brandeau/Douglas K. Owens/Eran Bendavid: The Cost-Effectiveness of Preexposure Prophylaxis for HIV Prevention in the United States in Men Who Have Sex With Men. In: Annals of Internal Medicine 156 (2012), H. 8, S. 541-550; Karen Schneider/Richard T. Gray/David P. Wilson: A Cost-Effectiveness Analysis of HIV Preexposure Prophylaxis for Men Who Have Sex With Men in Australia. In: Clinical Infectious Diseases 58 (2014), H. 7, S. 1027-1034; Valentina Cambiano/Alec Miners/David Dunn/Sheena McCormack/Koh Jun Ong/O Noel Gill/Anthony Nardone/Monica Deai/Nigel Field/Graham Hart/Valerie Delpech/Gus Caims/Alison Rodger/Andrew N. Phillips: Cost-effectiveness of pre-exposure prophylaxis for HIV prevention in men who have sex with men in the UK: a modelling study and health economic evaluation. In: The Lancet Infectious Diseases 18 (2018), H. 1, S. 85-94; Mingwang Shen/Yanni Xiao/Libin Rong/Lauren Ancel Meyers/Steven E. Bellan: The cost-effectiveness of oral HIV pre-exposure prophylaxis and early antiretroviral therapy in the presence of drug resistance among men who have sex with men in San Francisco. In: BMC Medicine 16 (2018), H. 1, S. 58.

${ }^{93}$ Vgl. George Ayala/Keletso Makofane/Glenn-Milo Santos/Jack Beck/Tri D. Do/Pato Herbert/Patrick A. Wilson/Thomas Pyun/Sony Arreola: Access to Basic HIV-Related Services and PrEP Acceptability among Men Who Have sex with Men Worldwide: Barriers, Facilitators, and Implications for Combination Prevention. In: Journal of Sexually Transmitted Diseases, 2013 (2013), 953123; Emily Land: PrEP and reaching people of color, trans women, and young people, 2017, https://betablog.org/prep-reaching-people-color-trans-women-young-people/ [letzter Zugriff am 15.8.2018]; Jae M. Sevelius/JoAnne Keatley/Nikki Calma/Emily Arnold: ,I am not a man': Trans-specific barriers and facilitators to PrEP acceptability among transgender women. In: Global Public Health 11 (2016), H. 7-8, S. 1060-1075; Latesha Elopre/Kachina Kudroff/Andrew O. Westfall/Edgar T. Overton/Michael J. Mugavero: Brief Report. The Right People, Right Places, and Right Practices: Disparities in PrEP Access Among African American Men, Women, and MSM in the Deep South. In: Journal of Acquired Immune Deficiency Syndromes 74 (2017), H. 1, S. 56-59; Kathleen R. Page/Omar Martinez/Karen Nieves-Lugo/Maria Cecilia Zea/Suzanne Dolwick Grieb/Thespina J. Yamanis/Kaitlin Spear/Wendy W. Davis: Promoting Pre-exposure Prophylaxis to Prevent HIV Infections Among Sexual and Gender Minority Hispanics/Latinxs. In: AIDS Education and Prevention 29 (2017), H. 5, S. 389-400; Villarosa (wie Anm. 3).
} 
Eigenverantwortung beruhen und schutzbedürftige Gruppen nicht ansprechen, weil sie keine Verankerung in den Communities haben und das Insistieren auf dem rationalen Verhalten deshalb als Versuch der Fremdbestimmung wahrgenommen werden kann. ${ }^{94}$ Darüber hinaus basiert die Einhaltung des Einnahmeregimes von PrEP auf der Identifikation als jemand, der Gefahr läuft, sich mit HIV zu infizieren. Diese Identifizierung ist bereits in schwulen Communitys, die seit mehr als drei Jahrzehnten mit HIV zu tun haben, mit Stigma behaftet - es ist also nicht verwunderlich, dass in Gemeinschaften oder Gruppen, in denen HIV kein Dauerthema ist - wie beispielsweise bei sich heterosexuell identifizierenden MSM -, eine solche Identifizierung noch schwieriger ist. Ein damit verbundenes Problem sind die oft immer noch enormen Kosten für HIV-Medikamente. Diese sind von Land zu Land unterschiedlich, da in manchen Ländern, unter anderem Deutschland, Generika eingesetzt werden, in anderen Ländern, wie den USA, die Patente dies noch verhindern. ${ }^{95}$ Grundsätzlich handeln Pharmaunternehmen im Gegensatz zum Interesse von Patienten, potenziellen PrEP-Anwendern und der Öffentlichkeit, wenn sie die rechtlichen Rahmenbedingungen und Patente zur Gewinnmaximierung kreativ ausnutzen. Dies ist umso skandalöser, wenn man bedenkt, dass die großen PrEP-Studien von öffentlichen Forschungseinrichtungen finanziert wurden. ${ }^{96}$

Die medizinische und allgemeine öffentliche Anti-PrEP-Perspektive kritisiert PrEP mit den zwei Argumenten, die ich oben schon diskutiert habe: dem Problem anderer STIs und dem Problem der Kosten. Darüber hinaus gibt es fünf weitere Argumente, die gegen PrEP in Medizin und Öffentlichkeit vorgebracht werden: Erstens wird angesichts möglicher Nebenwirkungen eine allgemeine Skepsis gegenüber der Idee geäußert, gesunde Körper zu behandeln; zweitens wird die Möglichkeit angeführt, dass sich Truvada-resistente HIV-Stämme entwickeln könnten; drittens das Problem der Arzneimittel-Compliance (Einhaltung der Behandlungsvorgaben); viertens spezifische institutionelle Grenzen in den öffentlichen Gesundheitssystemen, die ich am Beispiel Deutschlands veranschaulichen werde; und fünftens wird das homophobe Argument formuliert, dass die breite Öffentlichkeit nicht für das Vergnügen von Schwulen bezahlen sollte.

\footnotetext{
${ }^{94}$ Vgl. van Doorn (wie Anm. 47).

${ }^{95}$ Vgl. Tim Fitzsimons: Generic HIV prevention drug coming in 2020, Gilead says, 2019, https://www.nbcnews.com/feature/nbc-out/generic-hiv-prevention-drug-coming-2020-gilead-says-n1003391 [letzter Zugriff am 24.1.2020].

${ }^{96} \mathrm{Vgl}$. Summers (wie Anm. 61)..
} 
Viele Mediziner_innen, insbesondere, wenn sie keine HIV-Spezialist_innen sind, sind skeptisch gegenüber der Idee, gesunde Körper zu Präventionszwecken zu behandeln. Sie argumentieren, dass, obwohl Benutzer kaum Nebenwirkungen erfahren, Truvada immer noch ein starkes Medikament ist, das Niere, Leber und möglicherweise die Knochenintegrität angreift. Darüber hinaus ist es möglich, dass Truvada langfristige Nebenwirkungen hat, die noch unbekannt sind. ${ }^{97}$ Diese Einstellung zur Medikalisierung kann kulturell verankert sein. In Deutschland ist eine skeptische Haltung stärker verbreitet als in den USA. Die Einstellung der Öffentlichkeit in den USA zu Pharmazeutika und Biomedizintechnik kann als pragmatisch offen bezeichnet werden, während die Deutschen den (bio)technologischen Eingriffen in Körper und Natur eher skeptisch gegenüberstehen. ${ }^{98}$ Zweitens diskutieren Forscher_innen die Möglichkeit, dass sich Truvada-resistente HIV-Stämme entwickeln könnten und kontrollieren eine solche Entwicklung in Studien, aber bisher sind keine resistenten Stämme aufgetreten. ${ }^{99}$ Drittens ist eine zu geringe Medikamenteneinnahme ein Problem, auf das alle Studien hinweisen. Dies führt jedoch nicht zu vielen Infektionen, da Truvada auch bei niedrigen Adhärenzraten wirksam ist, so dass die Adhärenzniveaus im Allgemeinen hoch genug sind, um eine Prävention zu gewährleisten. ${ }^{100}$ Die Einhaltung der Behandlungsvorgaben bleibt jedoch ein entscheidender Faktor und muss durch PrEP-Programme angegangen werden.

Viertens ist eine Besonderheit des deutschen Gesundheitssystems, dass die gesetzliche Krankenversicherung in der Regel keine präventiven Arzneimittel abdeckt, weshalb PrEP lange nicht von den gesetzlichen Krankenkassen bezahlt wurde. Der Gemeinsame Bundesausschuss, das höchste Gremium der Selbstverwaltung im deutschen Gesundheitswesen, behauptete noch 2016 - entgegen den

\footnotetext{
${ }^{97}$ Vgl. Lauren V. Wood: Why I voted „no“ to Truvada PrEP. In: Annals of Internal Medicine 157 (2012), H. 7, S. 519-520.

${ }^{98}$ Vgl. Heiner Meulemann: Die Natur und das Leben: Naturalismus in Weltbildern und als Einstellung zur Biotechnik in der deutschen Bevölkerung. In: Wolfgang van den Daele (Hg.): Biopolitik (Leviathan, Sonderheft 23). Wiesbaden 2005, S. 155-175; Bettina Schöne-Seifert: Von der Medizin zur Humantechnologie? Ärztliches Handeln zwischen medizinischer Indikation und Patientenwunsch. In: van den Daele (Hg., wie Anm. 98), S. 179-199.

${ }^{99}$ Vgl. Constance Delaugerre/Christophe Rodriguez/Catherine Capitante/Marie-Laure Nere/Mélanie MercierDarty/Diane Carette/Gilles Pialoux/Laurent Cotte/Isabelle Charreaue/Jean-Michel Molina: Drug resistance among patients who acquired HIV infection in a preexposure prophylaxis trial. In: AIDS 32 (2018), H. 16, S. $2353-2361$. ${ }^{100}$ Vgl. Jessica E. Haberer: Current concepts for PrEP adherence in the PrEP revolution. From clinical trials to routine practice. In: Current Opinion in HIV and AIDS 11 (2016), H. 1, S. 10-17; Elizabeth F. Closson/Jennifer A. Mitty/Jowanna Malone/Kenneth H. Mayer/Matthew J. Mimiaga: Exploring strategies for PrEP adherence and dosing preferences in the context of sexualized recreational drug use among MSM: a qualitative study. In: AIDS Care 30 (2018), H. 2, S. 191-198.
} 
medizinischen Erkenntnissen -, dass verhaltensbasierte Prävention (Kondome) für jeden funktioniere und dass Prävention daher eine Frage der Eigenverantwortung sei. ${ }^{101}$ Eine Strategie der deutschen PrEP-Aktivist_innen war es, einzelne Krankenkassen mit der Begründung, dass dies ihre Popularität bei ihren Kund_innen erhöhen könnte, von einer Finanzierung der Medikamente zu überzeugen. In diesem Sinne bieten die deutschen Krankenkassen schon heute eine Vielzahl von Lifestyle- und Wellnessbehandlungen wie die Homöopathie an, die im Gegensatz zu PrEP nicht nachweislich wirksam sind. Rückblickend betrachtet war es aber die Entscheidung des schwulen Bundesgesundheitsministers Spahn (CDU), die dazu führte, dass die PrEP seit September 2019 von den gesetzlichen Kassen übernommen wird. ${ }^{102}$

Fünftens ist in der medizinischen Fachwelt und in der Öffentlichkeit eine homophobe Einstellung weit verbreitet. Stereotype von promiskuitiven schwulen Männern sind immer noch verbreitet und ihre Verbreitung ist in letzter Zeit durch den Einfluss von Rechtspopulisten in Europa und den Vereinigten Staaten gestiegen. ${ }^{103}$ PrEP wird mit einer Entscheidung für eine riskante Sexualität in Verbindung gebracht, die als unmoralisch angesehen wird. Die Kosten sollten diesem Argument nach nicht von der Allgemeinheit bezahlt. ${ }^{104}$ Diese homophobe Begründung, die die individuelle Verantwortung für die Prävention qua Verhalten betont, ignoriert die Tatsache, dass die HIV-Epidemie vor allem Schwule, Trans`_Personen und People of Color betrifft, die alle unterprivilegierte Minderheiten sind und öffentliche Unterstützung verdienen. Da das Ausmaß der HIV-Epidemie heute auf die eklatant homophobe Reaktion insbesondere in den USA ${ }^{105}$ in den 1980er Jahren

\footnotetext{
${ }^{101}$ Vgl. Deutsche Aidshilfe: HIV-Prophylaxe: Gemeinsamer Bundesausschuss erklärt sich für unzuständig, 2016, https://www.aidshilfe.de/meldung/hiv-prophylaxe-gemeinsamer-bundesausschuss-erklaert-unzustaendig [letzter Zugriff am 19.6.2018].

${ }^{102} \mathrm{Vgl}$. Bundesministerium für Gesundheit: Vorurteile abbauen, Neuansteckungen verhindern, 2018, https://www.bundesgesundheitsministerium.de/ministerium/meldungen/2018/juli/iac.html\#c13586 [letzter Zugriff am 10.1.2020].

103 Siehe Anm. 67.

${ }^{104}$ Zwei Beispiele für diesen weit verbreiteten homophoben Diskurs sind der Kommentar einer deutschen Lokalzeitung über die Ankündigung, dass die Kosten für PrEP von deutschen Krankenkassen übernommen werden, und die Benutzerkommentare eines älteren Artikels über PrEP auf der Mainstream-Newsseite „Spiegel Online“, vgl. Irene Berres: HIV-Schutz mit Medikamenten. „Die Leute können sich das nicht leisten“, 2017, http://www.spiegel.de/gesundheit/diagnose/hiv-schutz-mit-medikamenten-die-leute-koennen-sich-das-nicht-leisten-a1131570.html [letzter Zugriff am 15.9.2018]; Dennis Klein: Aids-Hilfe kritisiert „diskriminierenden“ Kommentar in Magdeburger „Volksstimme“, https://www.queer.de/detail.php?article_id=31603 [letzter Zugriff am 15.5.2018].

${ }^{105}$ In Deutschland war die HIV-Präventionsarbeit schon früh recht fortschrittlich, vgl. Michael Bochow: Hat AIDS die soziale Situation schwuler Männer verändert? In: Detlef Grumbach (Hg.): Was heißt hier schwul? Politik und Identität im Wandel. Hamburg 1997, S. 139-149.
} 
zurückzuführen ist (die in erster Linie das Ausbleiben einer Reaktion war, was zum Tod von Millionen führte), muss man sich über die Ebene individueller Verantwortung hinausbewegen und sich dem Ausgleich vergangener Ungerechtigkeiten zuwenden, indem man heute wirksame Präventionsprogramme anbietet.

\section{Die demokratische Biopolitik von PrEP}

Der Fall PrEP zeigt die Komplexität der zeitgenössischen Biopolitik. PrEP ist ein Fall der aktuellen Biopolitik, bei der Belange von Public Health, Konzeptualisierungen der Normalität und eine Ethik der Sexualität kombiniert werden, und ist gleichzeitig Teil eines größeren Trends zur Medikalisierung im Allgemeinen und zur Medikalisierung von Sex im Besonderen. ${ }^{106}$ Was bei PrEP besonders sichtbar ist, ist der Zusammenhang zwischen gemeinschaftlichen Sexualnormen, sexueller (medizinischer) Technologie und homophober Stigmatisierung. PrEP ist ein Dispositiv, das die sexuelle Subjektivierung verändert, und es ist vor allem diese Veränderung, die sowohl aus der Perspektive der schwulen Subjektivität als auch aus der Perspektive des medizinischen Fachpersonals umstritten ist und z.T. bekämpft wird.

Die Abbildung der Debatte zeigte, dass unterschiedliche biopolitische Ansätze spezifische Stärken und Schwächen haben, wenn sie auf PrEP angewendet werden. Die bestehende Literatur zur Biopolitik ist auf zwei Ebenen, normativ und analytisch, mit der aktuellen Debatte über PrEP verbunden: Die Theorie der Biopolitik kann eine Position gegenüber PrEP einnehmen und erklären, was in der Debatte über PrEP vor sich geht. Die beiden bewerteten biopolitischen Theorie-Schulen, Gouvernementalitätsstudien und Italienische Theorie, bieten, wenn sie auf PrEP angewendet werden, Argumente, die auch in der öffentlichen Debatte um PrEP vorgebracht werden. Eine ähnliche Position wie Deans Kritik an der repressiven Seite der „Pharmamacht“ und ihre Verbindung zu Kapitalinteressen findet sich in einigen kritischen Aussagen über PrEP, und Ailios Argument für die Ausweitung des Zugangs zur HIV-Behandlung unabhängig von subjektiven Rechten könnte die Forderung von HIV-Mediziner_innen nach einer besseren HIV-Medikamentenversorgung für gefährdete Communities unterstützen. In beiden Fällen werden jedoch nur oberflächliche theoretische

\footnotetext{
${ }^{106}$ Vgl. Cacchioni/Tiefer (wie Anm. 9).
} 
Elemente der Biopolitik verwendet, die die politische Debatte nicht voranbringen. Aus der Perspektive des PrEP-Aktivismus erscheint Deans Kritik weit hergeholt, steht im Verdacht derVerschwörungstheorie und wird den tatsächlichen Kämpfen nicht gerecht, während Ailios Forderung trivial ist und Espositos biopolitischen Überbau einer großen Erzählung der Moderne nicht gebraucht hätte.

Die differenzierte biopolitiktheoretische Darstellung, die von Rose und Rabinow vertreten wird und mit dem Forschungsfeld des Patient_innenaktivismus verbunden ist, bietet einen theoretischen Rahmen, um die Komplexität der Politik um PrEP besser zu verstehen. Die ökonomischen Interessen von Pharmaunternehmen, die diskursiven Verschiebungen in Richtung Medikalisierung im Gesundheitssektor und die Handlungsmacht von und komplexe Vernetzung zwischen Fachleuten, Anwender_innen, Patient_innen und Aktivist_innen werden dabei berücksichtigt. Rose kritisiert düstere Visionen einer überwältigenden biopolitischen und pharmazeutischen Macht, wie sie unter anderem von Dean entworfen wurden, und weist darauf hin, dass Biopolitik und die Implementierung neuer Medizintechnik komplexe politische Verhandlungen über somatische Ethik und viele verschiedene Akteure, die sich an biologischer Staatsbürgerschaft beteiligen, also aktiv die Politik aushandeln und mitbestimmen, beinhalten. Die politische Debatte um PrEP ist eine Instanziierung Fall solch komplexerVerhandlungen, die ich als demokratisch bezeichne: PrEP als demokratische Biopolitik. Die Debatte um PrEP so als demokratische Aushandlung zu bezeichnen, hat sowohl deskriptiven als auch normativen Charakter, das heißt einerseits werden die existierenden politischen Prozesse so besser beschrieben als etwa mit der quasi-verschwörungstheoretischen Position Deans, und zweitens kann man dadurch eine weitere Demokratisierung fordern. Fünf Komplexe sind für die Analyse und Kritik des demokratischen Gehalts der PrEP-Debatte zentral:

Erstens die Fragen der Repräsentation, der Macht und des Interesses, die auf dem Spiel stehen: Wer darf über wünschenswerte präventive Technologien entscheiden und wird dabei berücksichtig, wer in Verfahren eingebunden wird? Wer finanziert die Prävention und wer profitiert? Zweitens werden verschiedene sexuell-somatische Ethiken, d.h. die Möglichkeiten, die Sexualnormen von Gemeinschaften in Bezug auf medizinische Technologien zu gestalten, problematisiert und müssen verhandelt werden.Verschiedene ethische Entscheidungen kollidieren (z.B. für das Kondom vs. gegen das Kondom), und es sollten Kompromisse gefunden werden, weil die Hegemonie der einen Position die andere bedroht. Drittens müssen die Verhandlungen die Grenzen des demokratischen Diskurses 
berücksichtigen. Diese Grenzen werden in der Diskussion um PrEP oft verletzt, wenn homophobe und Slut-Shaming-Hassreden sowohl von schwulen als auch von nicht-schwulen Akteuren getätigt werden. Diese Hassrede ergibt sich nicht nur aus den Absichten des Einzelnen, sondern auch aus den sozialen Strukturen: Heteronormativität (die diskursive Hegemonie von Heterosexualität und Cis-Geschlechtlichkeit) und Homonormativität (die Unterscheidung zwischen guten und schlechten Schwulen). Daher ist politische Bildung und Antidiskriminierungsarbeit notwendig, um diese Einstellungen zu ändern und die Menschen auf den demokratischen Austausch über PrEP vorzubereiten. Dabei handelt es sich um die komplexe Verhandlung der Freiheit der Akteure und des potenziellen politischen Paternalismus, da politische Bildung selbst Normen kreiert. ${ }^{107}$ Viertens wirft PrEP Fragen nach der Rationalität legitimer Diskurse auf, da einerseits irrationale, medizinisch falsch informierte und hasserfüllte Rede eines der zentralen Probleme in der Debatte ist. Andererseits besteht ein großes Defizit bei der Öffentlichkeitsarbeit zur Prävention und bei sexuellen Kampagnen darin, dass sie sich oft auf ein rationalistisches Paradigma stützen, das davon ausgeht, dass mehr Informationen zu weniger riskantem Sex führten und dass Sexualpraktiken von bewussten und rationalen Akteuren kontrolliert werden. Erfolgreiche PrEP-Programme gehen vernünftigerweise (d.h. auf der Grundlage medizinischer Erkenntnisse) über dieses rationalistische Sex-Paradigma hinaus, indem sie die irrationalen Aspekte von Sex, d.h. Fantasie, Begehren und Subjektivität, berücksichtigen. Im Gegensatz zu den klassischen deliberativen Demokratietheorien, die Unvernunft ausschlieBen, macht die demokratische Biopolitik von PrEP die Unvernunft - sexuelles Begehren und Subjektivität - zum Ausgangspunkt der politischen Debatte. Die demokratische Biopolitik von PrEP bestätigt demokratische Theorien, die argumentieren, dass demokratische Vernunft von derVerarbeitung seines Anderen, des Unvernünftigen und Irrationalen, abhängt. ${ }^{108}$

PrEP als Problem der demokratischen Biopolitik zu betrachten, trägt nicht nur der Komplexität der laufenden Debatte Rechnung, sondern ermöglicht es auch, Wege zur weiteren Demokratisierung aufzuzeigen. Eine solche Demokratisierung erfordert die Erkenntnis, dass Begehren nicht gegeben, sondern ein Ergebnis der sexuellen Subjektivierung durch sexuell-somatische Ethik, ist, die von medizinischen Technologien und Programmen des Gesundheitswesens beeinflusst wird. Wenn diese Prozesse unbemerkt ablaufen, ist es schwierig, sie demokratisch zu verhandeln. Sie explizit zu ma-

\footnotetext{
107 Schubert: Freiheit (wie Anm. 24), S. 305-312.

${ }^{108}$ Vgl. Christoph Menke: Kritik der Rechte. Berlin 2015.
} 
chen, hilft, den demokratischen Diskurs zu fördern. Dies würde es auch ermöglichen, das zugrundeliegende Problem der Homophobie in großem Umfang durch politische Institutionen aus Bildung und Recht anzugehen, beispielsweise durch verbindliche queere Aufklärungsarbeit an Schulen und einen verbesserten Schutz gegen homophobe Beleidigungen und Übergriffe. Die Stärkung der Position der (potenziellen) PrEP-Nutzer_innen als wichtigster Interessengruppe in der Debatte durch ihre Einbeziehung in gesundheitspolitische Entscheidungen ist von großer Bedeutung - dass hier noch riesiges Demokratisierungspotential besteht, hat jüngst der Implementierungsprozess von PrEP in den deutschen gesetzlichen Krankenkassen gezeigt: Über die Regulierungen zur Medikamentenvergabe und zur notwendigen Fortbildung der Ärzte wurde völlig intransparent und undemokratisch, ohne Einbezug von Community oder Aktivist_innen entschieden. Wirkliche Demokratisierung der biopolitischen Sexualpolitik kann nicht ohne einen solchen Einbezug funktionieren. Schließlich würde Demokratisierung die Minimierung von Kosten im Gesundheitswesen und von Gewinnen von Pharmakonzernen bedeuten, um einen erschwinglichen Zugang zu PrEP für diejenigen zu schaffen, die sie benötigen. Dies erfordert nichts weniger als eine Revolution des globalen Patentrechts, das im Augenblick Kosten für die Forschung der Allgemeinheit auferlegt und Gewinne privatisiert.

Ich fasse zusammen: PrEP ist keine repressive Strategie der Biopolitik, die den ,Homosexuellen“, nachdem sie vor 100 Jahren vom medizinischen Diskurs als Kategorie erfunden und seitdem pathologisiert wurden, jetzt auch noch Pillen aufzwingt. Sondern PrEP kann beschrieben werden als demokratische Biopolitik, bei der verschiedene Akteure zusammenarbeiten und neue technischmedizinische und sexualethische Standards verhandeln. Diese Perspektive trägt dazu bei, die politischen Ambivalenzen und Gefahren, aber auch Potenziale von PrEP, insbesondere zur Destigmatisierung von schwulem Sex, zu beschreiben - und es vor allem zu kritisieren, wenn PrEP undemokratisch gesteuert wird und die weitere Demokratisierung dieser Prozesse zu fordern. 